\title{
Stable radiation-controlling boundary conditions for the generalized harmonic Einstein equations
}

\author{
O Rinne \\ Theoretical Astrophysics 130-33, California Institute of Technology, Pasadena, CA 91125, USA \\ E-mail: rinne@tapir.caltech.edu
}

Received 12 June 2006, in final form 6 September 2006

Published 6 October 2006

Online at stacks.iop.org/CQG/23/6275

\begin{abstract}
This paper is concerned with the initial-boundary value problem for the Einstein equations in a first-order generalized harmonic formulation. We impose boundary conditions that preserve the constraints and control the incoming gravitational radiation by prescribing data for the incoming fields of the Weyl tensor. High-frequency perturbations about any given spacetime (including a shift vector with a subluminal normal component) are analysed using the Fourier-Laplace technique. We show that the system is boundary stable. In addition, we develop a criterion that can be used to detect weak instabilities with polynomial time dependence, and we show that our system does not suffer from such instabilities. A numerical robust stability test supports our claim that the initial-boundary value problem is most likely to be well posed even if non-zero initial and source data are included.
\end{abstract}

PACS numbers: 04.25.Dm, 02.60.Lj, 04.20.Cv, 04.20.Ex

(Some figures in this article are in colour only in the electronic version)

\section{Introduction}

Most attempts to solve the Einstein equations numerically are based on the Cauchy, or $3+1$ formulation of general relativity, in which one foliates spacetime into three-dimensional spacelike hypersurfaces of constant time $t$. Initial data satisfying the constraint equations are prescribed on the $t=0$ surface and the evolution equations are integrated towards the future. Typically, one truncates the domain of integration and only aims to find solutions on a compact spatial manifold with artificial timelike boundaries, on which boundary conditions must be specified. These should satisfy a number of requirements as given below.

(i) The initial-boundary value problem (IBVP) should be well posed, i.e. for given initial and boundary data a unique solution should exist and it should depend continuously on the data. 
(ii) The boundary conditions must be compatible with the constraints in the sense that if the constraints are satisfied initially, then they remain satisfied at all times.

(iii) The boundary conditions should (in some sense) control the incoming gravitational radiation.

The last requirement is of particular importance if one aims to obtain reliable information about the gravitational radiation emitted by compact sources such as coalescing binary black holes. One way to describe the incoming radiation is in terms of the incoming fields of the evolution system that the Weyl tensor obeys by virtue of the Bianchi identies. These incoming fields are proportional to the Newman-Penrose scalar $\Psi_{0}$, a quantity that is invariant under infinitesimal coordinate transformations of Kerr spacetime. If the outer boundary is placed sufficiently far away from the source, then one expects

$$
\Psi_{0}=0
$$

to be a reasonable 'no-incoming-radiation' boundary condition. Conditions of this type have been considered by a number of authors [1-4].

Since the initial-boundary problem for Einstein's equations was first studied by Stewart [5] with numerical relativity in mind, this has been a very active field of research. However, there is currently only one formulation, due to Friedrich and Nagy [1], that satisfies all the above requirements for the fully nonlinear vacuum Einstein equations. It uses a tetrad formalism and evolves the components of the Weyl tensor as separate variables. All the boundary conditions are written in a maximally dissipative form, and well posedness of the IBVP follows from standard theorems for symmetric hyperbolic systems with such boundary conditions [6-8]. It would be desirable to obtain a similar result for a metric formulation of the Einstein equations which does not evolve the Weyl tensor separately, such as most of the formulations currently used in numerical relativity (see, however, [9] for an implementation of a tetrad-based formulation). In some cases, well posedness has been proved (at least partially) for boundary conditions that satisfy conditions (i) and (ii) but not (iii) (see for example [10-16], in particular harmonic formulations [17-20]). The major obstruction is the fact that the physical boundary conditions (1) as well as (in general) the constraint-preserving boundary conditions contain second rather than first derivatives of the metric (such boundary conditions are said to be of differential type). Hence, they are not in a maximally dissipative form and the standard theorems do not apply. Progress can still be made in a certain approximation (typically in the high-frequency limit or for linearizations about flat space) using FourierLaplace transform methods [21, 22]. This technique yields rather strong necessary conditions for well posedness, although sufficient conditions are not known for boundary conditions that are of a differential type. Applications to the Einstein equations and related problems can be found in [4, 5, 20, 23-25]. In particular, boundary conditions satisfying both requirements (ii) and (iii) were analysed in [4, 25]. Necessary conditions for well posedness were verified, but numerical experiments indicated that the systems still suffered from instabilities. Using rather different methods based on semigroup theory, Nagy and Sarbach [26] have recently proved well posedness of the IBVP (with radiation-controlling boundary conditions) for the linearized Einstein equations in the ADM formulation in a certain gauge. The gauge condition arises from a minimization principle and involves a fourth-order elliptic equation for the lapse function (which might be expensive to solve numerically).

In this paper, we consider a symmetric hyperbolic formulation of the Einstein equations based on generalized harmonic $(\mathrm{GH})$ coordinates (see [27] and references therein). Such coordinates $x^{a}$ obey the scalar wave equation

$$
\square x^{a}=H^{a}
$$


with a source $H^{a}$ that may depend on the coordinates and the spacetime metric $\psi_{a b}$ (but not on its derivatives). In this gauge, the Einstein equations reduce to a system of coupled nonlinear wave equations, with principal parts

$$
\square \psi_{a b} \simeq 0 \text {. }
$$

Recently, harmonic coordinates have played an important role in the first successful simulations of the inspiral, merger and ringdown of binary black holes by Pretorius [28, 29]. In [27], a first-order formulation of this system was derived, with particular emphasis on controlling the constraints in numerical evolutions. Boundary conditions satisfying requirements (ii) and (iii) were constructed, and a partial result on the well posedness of these boundary conditions was stated. The purpose of this paper is to elaborate on and generalize this result and to provide both analytical and numerical evidence that the system at hand is a strong candidate for a well-posed initial-boundary value formulation of the Einstein equations.

In section 2, we review the evolution equations and boundary conditions derived in [27]. The Fourier-Laplace analysis of the initial-boundary value problem is carried out in section 3. Whereas the well posedness result in [27] assumed that the shift vector was tangential at the boundary, we lift that restriction here and allow for an arbitrary shift. Because several characteristic fields propagate along the normal lines of the spacetime foliation, this may cause additional characteristic fields to be incoming, which complicates the analysis. Nevertheless, the result stated in [27] carries over to the general case. (We still assume for the analysis that the normal component of the shift is subluminal at the boundary, i.e. we disregard situations where either all the modes are outgoing or all the modes are ingoing. If all the modes are outgoing, e.g. inside a black hole, no boundary conditions are to be imposed. See section 5 and [27] for numerical tests of the GH evolution system involving this situation.)

The usual necessary condition for well posedness in the Fourier-Laplace framework amounts to verifying that a certain complex determinant has no zeros with a positive real part. We strengthen this result by showing that our system also obeys the Kreiss condition [21], which is stronger and implies that the solution can be estimated in terms of the boundary data. Related concepts of well posedness are briefly discussed. It has been claimed [4, 24] that even if the Kreiss condition is satisfied, weak instabilities with milder than exponential time dependence might exist if non-trivial initial data are included. In section 4, we show rather generally that the Kreiss condition excludes any instabilities with polynomial time dependence. We also demonstrate that for a bad choice of gauge boundary conditions that do not satisfy the Kreiss condition, a weak instability does exist. In order to supplement our analytical results, we perform a series of numerical robust stability tests in section 5. This involves adding random data to the initial and boundary conditions and to the right-hand sides of the evolution equations. The background spacetime is taken to be either flat space (including a shift) or Schwarzschild. A summary of the results and conclusions are given in section 6 .

\section{The initial-boundary value problem}

We begin by presenting the initial-boundary value problem for the Einstein equations in generalized harmonic gauge that is considered in this paper. This section follows closely [27]. Some additional details concerning the constraint-preserving boundary conditions in the case of a non-tangential shift at the boundary are provided.

\subsection{Evolution equations}

We consider the first-order formulation of the generalized harmonic evolution equations (3) derived in [27]. The fundamental variables are the spacetime metric $\psi_{a b}$ and its first derivatives 
$\Phi_{i a b} \equiv \partial_{i} \psi_{a b}$ and $\Pi_{a b} \equiv-t^{c} \partial_{c} \psi_{a b}$. Here, $t_{a} \propto \partial_{a} t$ denotes the unit timelike normal to the $t=$ const. hypersurfaces. The lower-case Latin indices from the beginning of the alphabet denote four-dimensional spacetime quantities, while the lower-case Latin indices from the middle of the alphabet are spatial.

The evolution equations take the form

$$
\begin{aligned}
& \partial_{t} \psi_{a b} \simeq\left(1+\gamma_{1}\right) N^{k} \partial_{k} \psi_{a b}, \\
& \partial_{t} \Pi_{a b} \simeq N^{k} \partial_{k} \Pi_{a b}-N g^{k i} \partial_{k} \Phi_{i a b}+\gamma_{1} \gamma_{2} N^{k} \partial_{k} \psi_{a b}, \\
& \partial_{t} \Phi_{i a b} \simeq N^{k} \partial_{k} \Phi_{i a b}-N \partial_{i} \Pi_{a b}+N \gamma_{2} \partial_{i} \psi_{a b},
\end{aligned}
$$

where $\simeq$ indicates that only the principal parts of the equations are displayed. The spatial metric $g_{i j}$, lapse function $N$ and shift vector $N^{i}$ are related to the 4-metric via the standard $\mathrm{ADM}$ form of the line element,

$$
\mathrm{d} s^{2}=\psi_{a b} \mathrm{~d} x^{a} \mathrm{~d} x^{b}=-N^{2} \mathrm{~d} t^{2}+g_{i j}\left(\mathrm{~d} x^{i}+N^{i} \mathrm{~d} t\right)\left(\mathrm{d} x^{j}+N^{j} \mathrm{~d} t\right) .
$$

From now on we will choose $\gamma_{1}=-1$, which ensures that the evolution equations are linearly degenerate. The parameter $\gamma_{2}$ was introduced in [27] in order to damp violations of the constraint

$$
\mathcal{C}_{i a b} \equiv \partial_{i} \psi_{a b}-\Phi_{i a b}=0
$$

There is also a parameter $\gamma_{0}$ hidden in the source terms, which is designed to damp violations of the generalized harmonic gauge constraint (2), based on a suggestion in [30]. It will play no role in this discussion.

The system (4)-(6) is symmetric hyperbolic for any choice of parameters $\gamma_{1}$ and $\gamma_{2}$. Hence, the Cauchy problem is well posed [22]. The characteristic variables and speeds in the direction of a unit spacelike vector $n_{i}$ are given by

$$
\begin{array}{lll}
u_{a b}^{0}=\psi_{a b}, & \text { speed } 0, \\
u_{a b}^{1 \pm}=\Pi_{a b} \pm \Phi_{n a b}-\gamma_{2} \psi_{a b}, & \text { speed }-N^{n} \pm N, \\
u_{A a b}^{2}=\Phi_{A a b}, & \text { speed }-N^{n},
\end{array}
$$

where here and in the following, an index $n$ denotes contraction with $n_{i}$ (e.g., $v_{n}=n_{i} v^{i}$ ) and the upper-case Latin indices denote projection with $P_{i j} \equiv g_{i j}-n_{i} n_{j}$ (e.g., $v_{A}=P_{A i} v^{i}$ ).

We consider a finite spatial domain of integration $\Omega$ with smooth boundary $\partial \Omega$. The spatial coordinate location of the boundary remains fixed in time, i.e. $\partial / \partial t$ is tangential to the three-dimensional timelike boundary $\partial \Omega \times[0, \infty)$. Boundary conditions have to be prescribed for the incoming characteristic fields, where now $n_{i}$ refers to the outward-pointing unit spacelike normal to $\partial \Omega$. Note that the number of incoming fields depends on the value of the normal component $N^{n}$ of the shift at the boundary. For $-N<N^{n} \leqslant 0$, only the fields $u_{a b}^{1-}$ are incoming; for $0<N^{n} \leqslant N$, the fields $u_{a b}^{1-}$ and $u_{A a b}^{2}$ are incoming.

\subsection{Constraint-preserving boundary conditions}

The boundary conditions should ensure that if the constraints vanish initially, then they vanish at all times. In other words, the subsidiary evolution system that the constraints obey as a consequence of the main evolution equations has to be well posed, with the unique solution being the trivial one. 
In our case, the primary constraints are the harmonic gauge constraint (2), which can be written in the form

$$
\mathcal{C}_{a} \equiv \psi^{b c} \Gamma_{a b c}+H_{a}=0,
$$

( $\Gamma_{a b c}$ being the Christoffel symbols of the metric $\psi_{a b}$ ), and the definition constraint $\mathcal{C}_{i a b}(8)$. If we define the first-order constraint variables

$$
\begin{aligned}
& \mathcal{C}_{i j a b}=2 \partial_{[i} \Phi_{j] a b}=2 \partial_{[i} \mathcal{C}_{j] a b}, \\
& \mathcal{F}_{a}=-t^{c} \partial_{c} \mathcal{C}_{a}+\cdots, \\
& \mathcal{C}_{i a}=\partial_{i} \mathcal{C}_{a}-g^{j k} \mathcal{C}_{i j k a}+\frac{1}{2} g_{a}{ }^{j} \psi^{c d} \mathcal{C}_{i j c d}+\cdots
\end{aligned}
$$

(omitting terms proportional to $\mathcal{C}_{i a b}$ ), then the constraint evolution system can be written in the simple form [27]

$$
\begin{aligned}
& \partial_{t} \mathcal{C}_{a} \simeq 0, \\
& \partial_{t} \mathcal{F}_{a} \simeq N^{i} \partial_{i} \mathcal{F}_{a}+N g^{i j} \partial_{i} \mathcal{C}_{j a}, \\
& \partial_{t} \mathcal{C}_{i a} \simeq N^{j} \partial_{j} \mathcal{C}_{i a}+N \partial_{i} \mathcal{F}_{a}, \\
& \partial_{t} \mathcal{C}_{i a b} \simeq 0 \\
& \partial_{t} \mathcal{C}_{i j a b} \simeq N^{k} \partial_{k} \mathcal{C}_{i j a b} .
\end{aligned}
$$

This system is clearly symmetric hyperbolic, and the characteristic variables and speeds are

$$
\begin{array}{lll}
c_{a}^{0 \pm}=\mathcal{F}_{a} \mp \mathcal{C}_{n a}, & \text { speed } & -N^{n} \pm N, \\
c_{a}^{1}=\mathcal{C}_{a}, & \text { speed } & 0, \\
c_{A a}^{2}=\mathcal{C}_{A a}, & \text { speed }-N^{n}, \\
c_{i a b}^{3}=\mathcal{C}_{i a b} & \text { speed } 0, \\
c_{i j a b}^{4}=\mathcal{C}_{i j a b}, & \text { speed }-N^{n} .
\end{array}
$$

Consider first the case $-N<N^{n} \leqslant 0$. The only incoming constraint fields are $c_{a}^{0-}$. We impose completely absorbing boundary conditions on them:

$$
c_{a}^{0-} \doteq 0
$$

( $\doteq$ denoting equality at the boundary). These can be translated into conditions on the normal derivatives of four of the main incoming fields $u_{a b}^{1-}$ by noting that

$$
\begin{aligned}
c_{a}^{0-} \simeq \sqrt{2}\left[k^{(c}\right. & \left.\psi^{d)}{ }_{a}-\frac{1}{2} k_{a} \psi^{c d}\right] \partial_{n} u_{c d}^{1-} \\
& +\frac{1}{2} P^{A}{ }_{a} \psi^{c d} \partial_{A} \Pi_{c d}-P^{A B} \partial_{A} \Pi_{B a}-P^{A B} t^{b} \partial_{A} \Phi_{B b a} \\
& +\frac{1}{2} t_{a} \psi^{c d} P^{A B} \partial_{A} \Phi_{B c d}+\gamma_{2}\left(P^{A B} \partial_{A} \psi_{B a}-\frac{1}{2} P^{A}{ }_{a} \psi^{c d} \partial_{A} \psi_{c d}\right) \\
& +P^{A B} \partial_{A} \Phi_{n B a}-\frac{1}{2} P^{A}{ }_{a} \psi^{c d} \partial_{A} \Phi_{n c d},
\end{aligned}
$$

where we have introduced the ingoing null vector $k^{a}=\left(t^{a}-n^{a}\right) / \sqrt{2}$.

In other words, the constraint-preserving boundary conditions imply boundary conditions on the normal derivatives of the following projection of $u_{a b}^{1-}$ :

$$
P_{a b}^{(\mathrm{C}) c d} u_{c d}^{1-} \equiv\left[\frac{1}{2} P_{a b} P^{c d}-2 l_{(a} P_{b)}{ }^{(c} k^{d)}+l_{a} l_{b} k^{c} k^{d}\right] u_{c d}^{1-},
$$


where we have also introduced the outgoing null vector $l^{a}=\left(t^{a}+n^{a}\right) / \sqrt{2}$. This projection has four degrees of freedom. Of the remaining six degrees of freedom of $u_{a b}^{1-}$, two will be fixed by the physical boundary conditions and four by the gauge boundary conditions.

Next, we consider the case $0<N^{n} \leqslant N$. Now the fields $c_{A a}^{2}$ and $c_{i j a b}^{4}$ are incoming as well. In addition to (26), we impose the boundary conditions

$$
0 \doteq c_{n A b c}^{4} \simeq \partial_{n} u_{A b c}^{2}-\partial_{A} \Phi_{n b c}
$$

which are conditions on the normal derivatives of the main characteristic fields $u_{A a b}^{2}$. The remaining incoming constraint fields, $c_{A a}^{2}$ and $c_{A B a b}^{4}$, also need boundary conditions. However, together with the physical and gauge boundary conditions, we have already used up all the incoming modes of the main evolution system and cannot impose any further boundary conditions actively. Fortunately, it turns out that $c_{A a}^{2} \doteq 0$ and $c_{A B a b}^{4} \doteq 0$ as a consequence of the constraint-preserving boundary conditions we have already imposed and the evolution equations. We prove this important point in the appendix, using the Fourier-Laplace method (we only consider the limit of high-frequency perturbations about a fixed background spacetime in that part of the analysis).

To summarize, we have imposed homogeneous maximally dissipative boundary conditions for the constraint evolution system. Because of general theorems for quasilinear symmetric hyperbolic systems with such boundary conditions [6-8], it follows that the constraint evolution system is well posed and the unique solution is the trivial one.

Setting to zero the incoming fields of the constraint evolution system at the boundary as in (26) is the standard prescription used in most works on constraint-preserving boundary conditions (e.g. $[3,5,10,11,14])$. This ensures that any constraint violations generated in a numerical evolution leave the computational domain without reflections (at least for normal incidence). In harmonic formulations, one sometimes considers the simpler alternative $\mathcal{C}_{a} \doteq 0$ [20], which does not involve any derivatives of the fundamental fields. These Dirichlet boundary conditions are clearly also consistent with the constraints, but they constitute a reflective boundary condition for the constraint evolution system (16)-(20). Numerical tests of such boundary conditions for an axisymmetric version [31] of the Z4 system [32] (which is very similar [30] to the generalized harmonic evolution system considered here) indicate that they can cause significant reflections of constraint violations [25].

\subsection{Physical boundary conditions}

The physical boundary conditions used in [27] are designed to control the gravitational radiation entering the domain through the boundary, following the prescription used in [1-4]. As explained in the introduction, the incoming radiation can be described in terms of the ingoing characteristic fields $w_{a b}^{-}$of the Weyl tensor evolution system:

$$
w_{a b}^{-}=2\left(P_{a}^{c} P_{b}{ }^{d}-\frac{1}{2} P_{a b} P^{c d}\right) k^{e} k^{f} C_{c e d f} .
$$

(The fields $w_{a b}^{-}$are proportional to the Newman-Penrose scalar $\Psi_{0}$ for a null tetrad containing the null vectors $k^{a}$ and $l^{a}$ defined above.) As a boundary condition, we impose

$$
w_{a b}^{-} \doteq \partial_{t} h_{a b}^{(\mathrm{P})} \text {. }
$$

The data $h_{a b}^{(\mathrm{P})}$ may be used to inject a gravitational wave through the boundary, for instance. Note that the expression for $w_{a b}^{-}$is only unique up to multiples of the constraints $\mathcal{C}_{i j a b}$ related to the index ordering in the first-order reduction. For a particular choice of index ordering, we can write $w_{a b}^{-}$in the form

$$
\begin{gathered}
w_{a b}^{-} \simeq\left(P_{a}{ }^{A} P_{b}{ }^{B}-\frac{1}{2} P_{a b} P^{A B}\right)\left[\partial_{n}\left(u_{A B}^{1-}+\gamma_{2} u_{A B}^{0}\right)+2 g^{i j} \partial_{A} \Phi_{i j B}-g^{i j} \partial_{A} \Phi_{B i j}\right. \\
\left.-P^{C D} \partial_{C} \Phi_{D A B}+t^{c} \partial_{A} \Phi_{n B c}-\partial_{A} \Pi_{B n}-t^{c} \partial_{A} \Phi_{B n c}\right] .
\end{gathered}
$$


Hence, (31) provides a boundary condition on the normal derivatives of

$$
P_{a b}^{(\mathrm{P}) c d} u_{c d}^{1-} \equiv\left(P_{a}^{c} P_{b}{ }^{d}-\frac{1}{2} P_{a b} P^{c d}\right) u_{c d}^{1-} .
$$

In [33], a hierarchy of boundary conditions has recently been developed that are perfectly absorbing for linearized gravitational radiation up to some arbitrary angular momentum number $L$. They can be viewed as successive improvements of the $\Psi_{0} \doteq 0$ boundary conditions and take the form

$$
\left.\partial_{t}\left(r^{2} l^{a} \partial_{a}\right)^{L-1}\left(r^{5} \Psi_{0}\right)\right|_{r=R}=0,
$$

where $r$ is an areal radial coordinate and it is assumed that the boundary is a sphere of radius $R$. We remark here that the stability result derived in this paper carries over to these improved boundary conditions (cf section 3.2).

\subsection{Gauge boundary conditions}

The remaining components of $u_{a b}^{1-}$ correspond to gauge degrees of freedom. To understand this, one should note that the generalized harmonic condition (2) does not fix the coordinates completely; there is still a remaining gauge freedom $x^{a} \rightarrow x^{a}+\xi^{a}$ for infinitesimal coordinate displacements $\xi^{a}$ that satisfy the wave equation. We may exploit this gauge freedom in order to choose the four remaining boundary conditions in any way we like. Our viewpoint here is that the gauge boundary conditions should guarantee that the IBVP is well posed, while still admitting arbitrary boundary data in order to be able to impose a variety of gauge conditions.

The simplest way appears to prescribe data $h_{a b}^{(\mathrm{G})}$ for those components of $u_{a b}^{1-}$ that are not specified by the constraint-preserving and physical boundary conditions,

$$
\begin{aligned}
P_{a b}^{(\mathrm{G}) c d} u_{c d}^{1-} & \equiv\left[\delta_{(a}^{c} \delta_{b)}^{d}-P_{a b}^{(\mathrm{C}) c d}-P_{a b}^{(\mathrm{P}) c d}\right] u_{c d}^{1-} \\
& =\left[2 l_{(a} k_{b)} l^{c} k^{d}+k_{a} k_{b} l^{c} l^{d}-2 k_{(a} P_{b)}{ }^{c} l^{d}\right] u_{c d}^{1-} \doteq h_{a b}^{(\mathrm{G})} .
\end{aligned}
$$

Equivalently, we can write (35) in the simpler form

$$
l^{b} u_{a b}^{1-} \doteq h_{a}^{(\mathrm{G})} .
$$

These (with $h^{(\mathrm{G})}=0$ ) are the gauge boundary conditions used in the numerical implementation of [27].

For illustrational purposes only, we shall also consider the following alternative set of gauge boundary conditions, which will turn out to be ill posed (to varying extent):

$$
t^{b} \Pi_{a b} \doteq h_{a}^{\left(\mathrm{G}^{\prime}\right)} .
$$

To linear order, these amount to prescribing (the time derivative of ) the lapse and shift at the boundary.

\section{Fourier-Laplace analysis}

In this section, we discuss necessary conditions for well posedness using the Fourier-Laplace technique. We begin with a review of the general theory, mainly following [5, 21] and highlighting some open questions. We then apply it to the generalized harmonic evolution system for the case of high-frequency perturbations about any arbitrary spacetime. 


\subsection{General theory}

Consider a linear symmetric hyperbolic system of evolution equations

$$
\partial_{t} \boldsymbol{u}=A^{i} \partial_{i} \boldsymbol{u},
$$

where $\boldsymbol{u}$ is an $m$-dimensional vector, $A^{i}$ are $m \times m$ constant symmetric matrices and $1 \leqslant i \leqslant n$. We take the spatial domain of integration to be the quarter-space $t \geqslant 0, x^{1} \geqslant 0,-\infty<$ $x^{2}, x^{3}, \ldots, x^{n}<\infty$. Initial data are prescribed at $t=0$,

$$
\boldsymbol{u}\left(0, x^{i}\right)=\boldsymbol{f}\left(x^{i}\right) .
$$

Let $l$ denote the number of negative eigenvalues of $A^{1}$ (i.e. the number of incoming modes). We impose boundary conditions at $x^{1}=0$ of the form

$$
S^{i} \partial_{i} \boldsymbol{u} \doteq \partial_{t} \boldsymbol{h},
$$

where $S^{i}$ are constant $l \times m$ matrices and $\boldsymbol{h}$ is a vector of boundary data (this non-standard form of the boundary conditions is required for our application in section 3.2).

We allow for the boundary to be (uniformly) characteristic, which is the case for most applications in physics, including the system discussed in this paper. After a suitable orthogonal transformation of the variables, we may write

$$
A^{1}=\left(\begin{array}{cc}
\mathbb{O}_{k} & 0 \\
0 & A
\end{array}\right),
$$

where $\mathbb{O}_{k}$ is the $k \times k$ zero matrix and $A$ is an $(m-k) \times(m-k)$ matrix. Similarly, we split

$$
\boldsymbol{u}=(\boldsymbol{z}, \boldsymbol{v})^{T} \text {. }
$$

The initial-boundary value problem (38)-(40) can be solved by means of a Laplace transform with respect to time and a Fourier transformation with respect to the spatial coordinates $x^{A}$ tangent to the boundary $(2 \leqslant A \leqslant n)$, i.e. we write $\boldsymbol{u}$ as a superposition of modes

$$
\boldsymbol{u}\left(t, x^{i}\right)=\tilde{\boldsymbol{u}}\left(x^{1}\right) \exp \left(s t+\mathrm{i} \omega_{A} x^{A}\right)
$$

with $s \in \mathbb{C}, \operatorname{Re}(s)>0$ and $\omega_{A} \in \mathbb{R}$. By inserting (43) into the evolution equations (38), we obtain the Fourier-Laplace-transformed version

$$
s \tilde{\boldsymbol{u}}=A^{1} \partial_{1} \tilde{\boldsymbol{u}}+\mathrm{i} \omega_{A} A^{A} \tilde{\boldsymbol{u}} .
$$

Partitioning the matrix

$$
s \mathbb{I}_{m}-\mathrm{i} \omega_{A} A^{A} \equiv B=\left(\begin{array}{cc}
B_{11} & B_{12} \\
B_{12}^{T} & B_{22}
\end{array}\right)
$$

in the same fashion as in (41) $\left(\mathbb{I}_{m}\right.$ being the $m \times m$ unit matrix), equation (44) splits into

$$
\begin{aligned}
& 0=B_{11} \tilde{\boldsymbol{z}}+B_{12} \tilde{\boldsymbol{v}}, \\
& A \partial_{1} \tilde{\boldsymbol{v}}=B_{12}{ }^{T} \tilde{\boldsymbol{z}}+B_{22} \tilde{\boldsymbol{v}} .
\end{aligned}
$$

Because $A^{i}$ are symmetric, the matrix $B$ and in particular $B_{11}$ in (45) has only non-zero eigenvalues. Hence, we may use the algebraic relations (46) in order to eliminate $\tilde{z}$ in favour of $\tilde{\boldsymbol{v}}$. We are left with the system of ordinary differential equations (ODEs)

$$
\partial_{1} \tilde{\boldsymbol{v}}=M \tilde{\boldsymbol{v}}, \quad M \equiv A^{-1}\left(B_{22}-B_{12}{ }^{T} B_{11}{ }^{-1} B_{12}\right) .
$$

A simple argument [21,34] shows that $M$ has precisely $l$ eigenvalues $\lambda_{1}, \lambda_{2}, \ldots, \lambda_{l}$ with a negative real part. Let $\boldsymbol{w}_{1}, \boldsymbol{w}_{2}, \ldots, \boldsymbol{w}_{l}$ denote the corresponding eigenvectors. Then, the 
general $L^{2}$ solution of (48) is given by

$$
\tilde{\boldsymbol{v}}\left(s, x^{1}, \omega\right)=\sum_{j=1}^{l} \sigma_{j} \boldsymbol{w}_{j}(s, \omega) \exp \left(\lambda_{j} x^{1}\right)
$$

with complex integration constants $\sigma_{j}$. (If the eigenvectors do not span the space, then some of $\sigma_{j}$ have to be replaced with polynomials in $x^{1}$-this will occur as a special case in our application.)

The constants $\sigma_{j}$ are determined by the boundary conditions: substituting (49) into the Fourier-Laplace transform of (40),

$$
S^{1} \partial_{1} \tilde{\boldsymbol{u}}+\mathrm{i} \omega_{A} S^{A} \tilde{\boldsymbol{u}} \doteq s \tilde{\boldsymbol{h}},
$$

we obtain a system of linear equations

$$
C(s, \omega) \boldsymbol{\sigma}=\tilde{\boldsymbol{h}},
$$

where $C$ is an $l \times l$ matrix.

Consider first the homogeneous boundary conditions, $\tilde{h}=0$. Suppose that $\operatorname{det} C=0$ for some $s$ with $\operatorname{Re}(s)>0$. Then (51) has a non-trivial solution, and hence the IBVP (38)-(40) with homogeneous boundary conditions has a non-trivial solution of the form (43). Now observe that with (43),

$$
\boldsymbol{u}\left(t, x^{i}\right)=\tilde{\boldsymbol{u}}\left(\alpha x^{1}\right) \exp \left(\alpha s t+\mathrm{i} \alpha \omega_{A} x^{A}\right)
$$

is also a solution, for any $\alpha>0$. Thus, we can find solutions which grow exponentially at an arbitrarily fast rate, and the IBVP is ill posed. Such solutions are called strong instabilities. (In a numerical simulation, $\alpha$ is determined by the highest frequency that can be represented on the grid. Hence, the growth rate of the instability increases with resolution.) We conclude that the determinant condition

$$
\operatorname{det} C(s, \omega) \neq 0 \quad \text { for } \quad \operatorname{Re}(s)>0
$$

is a necessary condition for well posedness.

Next, we consider the inhomogeneous boundary conditions (40). Formally, we can solve (51) for the integration constants $\sigma$ provided that the determinant condition is satisfied. What remains to be shown is that the solution (49) can be bounded in terms of the boundary data,

$$
|\tilde{\boldsymbol{v}}(s, 0, \omega)| \leqslant K|\tilde{\boldsymbol{h}}(s, \omega)|,
$$

with a constant $K>0$ that is independent of $s$ and $\omega$. This is known as the Kreiss condition [21]. Provided that the eigenvectors $\boldsymbol{w}_{i}$ in (49) are normalized in such a way that they remain finite as $\operatorname{Re}(s) \downarrow 0$ and as $|s| \rightarrow \infty,(54)$ is equivalent to

$$
\operatorname{det} C(s, \omega) \neq 0 \quad \text { for } \operatorname{Re}(s) \geqslant 0 \text {. }
$$

Comparing this with (53), the additional requirement is that there be no zeros $s$ of $\operatorname{det} C$ with $\operatorname{Re}(s)=0$. Such zeros are known as generalized eigenvalues.

If the Kreiss condition is satisfied, then it follows immediately that the IBVP (38), (40) is well posed for vanishing initial data. The unique solution is given by the inverse of the Fourier-Laplace transform, which is well defined because of the bound (54). In particular, we obtain an estimate of the form

$$
\int_{0}^{t}\|\boldsymbol{u}(\cdot, \tau)\|_{\Omega}^{2} \mathrm{~d} \tau \leqslant K_{T} \int_{0}^{t}\|\boldsymbol{h}(\cdot, \tau)\|_{\partial \Omega}^{2} \mathrm{~d} \tau
$$

in every time interval $0 \leqslant t \leqslant T$, where the constant $K_{T}$ is independent of the data $\boldsymbol{h}$ (the norms are $L^{2}$ norms over the half-space and the boundary, respectively). We say that the 
system is boundary stable [20]. It is this definition of stability that we shall prove for the generalized harmonic evolution system (section 3.2).

Closely related to boundary stability is the concept of strong well posedness in the generalized sense [20-22]. This requires that if we add a source term $\boldsymbol{F}\left(t, x^{i}\right)$ to the righthand side of the evolution equations (38), the following estimate holds:

$$
\int_{0}^{t}\|\boldsymbol{u}(\cdot, \tau)\|_{\Omega}^{2} \mathrm{~d} \tau+\int_{0}^{t}\|\boldsymbol{v}(\cdot, \tau)\|_{\partial \Omega}^{2} \mathrm{~d} \tau \leqslant K_{T}\left(\int_{0}^{t}\|\boldsymbol{h}(\cdot, \tau)\|_{\partial \Omega}^{2} \mathrm{~d} \tau+\int_{0}^{t}\|\boldsymbol{F}(\cdot, \tau)\|_{\Omega}^{2} \mathrm{~d} \tau\right)
$$

(recall that $\boldsymbol{v}$ refers to the modes with non-zero speeds). The crucial ingredient in proving (57) given (56) is the construction of a symmetrizer [20, 34, 35]. In doing this, it is usually assumed that the boundary conditions are in a maximally dissipative form

$$
v^{-} \doteq S v^{+}+g
$$

where $\boldsymbol{v}^{-}$and $\boldsymbol{v}^{+}$are the ingoing and outgoing (non-zero speed) modes, respectively, and $S$ is a (for our purposes constant) matrix. The symmetrizer method does not appear to be applicable if the boundary conditions are of the differential type (40).

So far we have assumed that the initial data $f$ vanish. One can always treat the case of general initial data by considering e.g. $\boldsymbol{u}^{\prime} \equiv \boldsymbol{u}-\mathrm{e}^{-t} \boldsymbol{f}$ so that the problem for $\boldsymbol{u}^{\prime}$ has zero initial data. However, in the evolution equations for $\boldsymbol{u}^{\prime}$, there will be an additional source term containing derivatives of $\boldsymbol{f}$, which will appear in the estimate generalizing (57). What one would like instead is an estimate of the form

$$
\begin{aligned}
\int_{0}^{t}\|\boldsymbol{u}(\cdot, \tau)\|_{\Omega}^{2} \mathrm{~d} \tau & +\int_{0}^{t}\|\boldsymbol{v}(\cdot, \tau)\|_{\partial \Omega}^{2} \mathrm{~d} \tau \\
& \leqslant K_{T}\left(\|\boldsymbol{f}(\cdot)\|_{\Omega}^{2}+\int_{0}^{t}\|\boldsymbol{h}(\cdot, \tau)\|_{\partial \Omega}^{2} \mathrm{~d} \tau+\int_{0}^{t}\|\boldsymbol{F}(\cdot, \tau)\|_{\Omega}^{2} \mathrm{~d} \tau\right),
\end{aligned}
$$

without any derivatives of $f$. This is referred to as strong well posedness. Majda and Osher [34] showed in the present case of a uniformly characteristic boundary that the Kreiss condition is also sufficient for strong well posedness, provided that the boundary conditions are maximally dissipative. However, nothing is known in general for boundary conditions of the form (40). In section 4, we will argue that the Kreiss condition is still useful in order to rule out certain weak instabilities that have a polynomial time dependence.

We close this section with some remarks on the scope of the Fourier-Laplace method. Technically, one can only apply Fourier and Laplace transforms if the evolution equations and boundary conditions have constant coefficients. However, well posedness is a concept that is associated with the behaviour of the high-frequency components of the solution. In many cases, one can show that a problem with variable coefficients is strongly well posed if all problems obtained by freezing the coefficients are strongly well posed ([22, theorem 8.4.9], see [36] for the extension from strictly hyperbolic to symmetric hyperbolic systems, see also the discussion in [20]). Strong well posedness can be further extended from linear to quasilinear systems (such as the Einstein equations), in which case the estimates will in general only hold in a finite time interval (see for example [22, section 8.5], [7, 8]). More general spatial domains $\Omega$ than the half-space can easily be treated by splitting $\partial \Omega$ into a finite number of portions, each of which can be smoothly mapped to the half-space [21, section 9.6.2]. One should note, however, that the proofs of the above extensions usually assume the boundary conditions to be maximally dissipative- the situation is much less clear for differential boundary conditions. 


\subsection{Application to the GH system}

In this section, we apply the Fourier-Laplace technique to the generalized harmonic system of section 2. For previous applications of this method to various formulations of the Einstein equations, see e.g. [4, 5, 20, 23-25].

First, we construct the most general frozen-coefficient problem by considering the limit of high-frequency perturbations of an arbitrary fixed background spacetime. Consider a point $p$ at the boundary. By rescaling and rotating the spatial coordinates if necessary, we can achieve that the 3-metric at $p$ is $g_{i j}=\delta_{i j}$, and by rescaling the time coordinate, we may also assume that the lapse at $p$ is $N=1$. However, we have to allow for an arbitrary shift vector. (If we performed a coordinate transformation that affected the shift vector, we would have to consider a moving boundary.) Furthermore, we may assume that the boundary is located at $x^{1} \equiv x=0$ and that the domain of integration is $x>0$.

Hence, the evolution equations (4)-(6) become

$$
\begin{aligned}
& \hat{\partial}_{t} \psi_{a b}=N^{A} \partial_{A} \psi_{a b}, \\
& \hat{\partial}_{t} \Pi_{a b}=N^{x} \partial_{x} \Pi_{a b}-\partial^{k} \Phi_{k a b}-\gamma_{2} N^{k} \partial_{k} \psi_{a b}, \\
& \hat{\partial}_{t} \Phi_{j a b}=N^{x} \partial_{x} \Phi_{j a b}-\partial_{j} \Pi_{a b}+\gamma_{2} \partial_{j} \psi_{a b},
\end{aligned}
$$

where we have introduced the operator $\hat{\partial}_{t} \equiv \partial_{t}-N^{A} \partial_{A}, N^{i}$ refers to the (constant) background shift vector, and it is now understood that spatial indices are raised and lowered with the unit metric.

We consider an ansatz similar to (43),

$$
\boldsymbol{u}\left(t, x^{i}\right)=\tilde{\boldsymbol{u}}(x) \exp \left[s t+\mathrm{i} \omega_{A}\left(x^{A}+N^{A} t\right)\right]
$$

for all the dependent variables $\boldsymbol{u}=\left\{\psi_{a b}, \Pi_{a b}, \Phi_{i a b}\right\}$, where $s \in \mathbb{C}$ with $\operatorname{Re}(s)>0$ and $x^{A}=\{y, z\}$. Because of the rotational invariance of the evolution equations and boundary conditions, we may without loss of generality take $\omega_{A}=\omega \delta_{A}{ }^{y}$, say, with $\omega>0$ (note that $\omega=0$ can be excluded because we have taken the high-frequency limit). It is convenient to eliminate $\omega$ from the following equations by defining $\xi \equiv \omega x$ and $\zeta \equiv s / \omega$.

Let us first consider the case $N^{x}=0$. Inserting the ansatz (63) into the evolution equations (60)-(62), we first obtain the algebraic relations

$$
\begin{aligned}
& \zeta \tilde{\psi}_{a b}=\mathrm{i} N^{y} \tilde{\psi}_{a b}, \\
& \zeta \tilde{\Phi}_{y a b}=-\mathrm{i} \tilde{\Pi}_{a b}, \\
& \zeta \tilde{\Phi}_{z a b}=0 .
\end{aligned}
$$

From these we deduce that $\tilde{\psi}_{a b}=\tilde{\Phi}_{z a b}=0$, and we eliminate $\tilde{\Phi}_{y a b}$ in favour of $\tilde{\Pi}_{a b}$. The remaining two evolution equations are turned into the ODE system

$$
\partial_{\xi}\left(\begin{array}{c}
\tilde{\Pi}_{a b} \\
\tilde{\Phi}_{x a b}
\end{array}\right)=\left(\begin{array}{cc}
0 & -\zeta \\
-\zeta-\frac{1}{\zeta} & 0
\end{array}\right)\left(\begin{array}{c}
\tilde{\Pi}_{a b} \\
\tilde{\Phi}_{x a b}
\end{array}\right) .
$$

The matrix has eigenvalues

$$
\lambda_{1}^{ \pm}= \pm \sqrt{1+\zeta^{2}}
$$

(the branch of the square root is chosen such that $\operatorname{Re}\left(\lambda_{1}^{+}\right)>0$ for $\operatorname{Re}(\zeta)>0$ ). Since we are only interested in $L^{2}$ solutions, i.e. solutions that decay as $x \rightarrow \infty$, we have to pick the 
eigenvalue $\lambda_{1}^{-}$. The corresponding eigenvector is $\left(\zeta,-\lambda_{1}^{-}\right)^{T}$ and hence the general $L^{2}$ solution is

$$
\left(\begin{array}{c}
\tilde{\Pi}_{a b} \\
\tilde{\Phi}_{x a b} \\
\tilde{\Phi}_{y a b} \\
\tilde{\Phi}_{z a b}
\end{array}\right)=\sigma_{1 a b} \mathrm{e}^{\lambda_{1}^{-} \xi} K_{1}^{-1}\left(\begin{array}{c}
\zeta \\
-\lambda_{1}^{-} \\
-\mathrm{i} \\
0
\end{array}\right)
$$

with arbitrary complex constants $\sigma_{1 a b}$. The normalization constant $K_{1}$ (and all similar constants in the following) is equal to the norm of the vector immediately following it.

Next, we discuss the case $\beta \equiv N^{x} \neq 0$. We obtain $\tilde{\psi}_{a b}=0$ as before, but now the ODE system reads

$$
\partial_{\xi}\left(\begin{array}{c}
\tilde{\Pi}_{a b} \\
\tilde{\Phi}_{x a b} \\
\tilde{\Phi}_{y a b} \\
\tilde{\Phi}_{z a b}
\end{array}\right)=\left(\begin{array}{cccc}
-\gamma^{2} \beta \zeta & -\gamma^{2} \zeta & -\mathrm{i} \gamma^{2} \beta & 0 \\
-\gamma^{2} \zeta & -\gamma^{2} \beta \zeta & -\mathrm{i} \gamma^{2} & 0 \\
\mathrm{i} / \beta & 0 & \zeta / \beta & 0 \\
0 & 0 & 0 & \zeta / \beta
\end{array}\right)\left(\begin{array}{c}
\tilde{\Pi}_{a b} \\
\tilde{\Phi}_{x a b} \\
\tilde{\Phi}_{y a b} \\
\tilde{\Phi}_{z a b}
\end{array}\right)
$$

where we have introduced the shorthand $\gamma^{2} \equiv\left(1-\beta^{2}\right)^{-1}$. The eigenvalues are

$$
\lambda_{1}^{ \pm}=\gamma^{2}\left(-\beta \zeta \pm \sqrt{\zeta^{2}+\gamma^{-2}}\right), \quad \lambda_{2}=\frac{\zeta}{\beta} .
$$

For $\beta>0$, only $\lambda_{1}^{-}$has a negative real part (recall that we are assuming $|\beta|<1$ ) and the corresponding solution is

$$
\left(\begin{array}{c}
\tilde{\Pi}_{a b} \\
\tilde{\Phi}_{x a b} \\
\tilde{\Phi}_{y a b} \\
\tilde{\Phi}_{z a b}
\end{array}\right)=\sigma_{1 a b} \mathrm{e}^{\lambda_{1}^{-} \xi} K_{1}^{-1}\left(\begin{array}{c}
\zeta-\beta \lambda_{1}^{-} \\
-\lambda_{1}^{-} \\
-\mathrm{i} \\
0
\end{array}\right)
$$

One observes that this contains the above result (69) for $\beta=0$ in a regular way so that we do not need to discuss these two cases separately in the following.

For $\beta<0$, the eigenvalue $\lambda_{2}$ also has a negative real part and hence the general $L^{2}$ solution is

$$
\left(\begin{array}{c}
\tilde{\Pi}_{a b} \\
\tilde{\Phi}_{x a b} \\
\tilde{\Phi}_{y a b} \\
\tilde{\Phi}_{z a b}
\end{array}\right)=\sigma_{1 a b} \mathrm{e}^{\lambda_{1}^{-} \xi} K_{1}^{-1}\left(\begin{array}{c}
\zeta-\beta \lambda_{1}^{-} \\
-\lambda_{1}^{-} \\
-\mathrm{i} \\
0
\end{array}\right)+\mathrm{e}^{\lambda_{2} \xi}\left[\sigma_{2 a b} K_{2}^{-1}\left(\begin{array}{c}
0 \\
-\mathrm{i} \beta \\
\zeta \\
0
\end{array}\right)+\sigma_{3 a b}\left(\begin{array}{l}
0 \\
0 \\
0 \\
1
\end{array}\right)\right] .
$$

More care is needed if $\zeta=-\beta$ because in that case, $\lambda_{1}^{-}=\lambda_{2}=-1$ and the corresponding eigenvectors are degenerate (this is the only case in which such a situation occurs). The most general solution is now of the form

$$
\left(\begin{array}{c}
\tilde{\Pi}_{a b} \\
\tilde{\Phi}_{x a b} \\
\tilde{\Phi}_{y a b} \\
\tilde{\Phi}_{z a b}
\end{array}\right)=\mathrm{e}^{-\xi}\left[\sigma_{1 a b} K_{1}^{-1}\left(\begin{array}{c}
-\beta \\
0 \\
\mathrm{i} \\
0
\end{array}\right)+\left(\sigma_{1 a b} \xi+\sigma_{2 a b}\right) K_{2}^{-1}\left(\begin{array}{c}
0 \\
1 \\
-\mathrm{i} \\
0
\end{array}\right)+\sigma_{3 a b}\left(\begin{array}{l}
0 \\
0 \\
0 \\
1
\end{array}\right)\right] \text {. }
$$

In order to work out the Fourier-Laplace transform of the boundary conditions, it is useful to note that in our set-up, $\left\{t^{a},(\partial / \partial x)^{a}=-n^{a},(\partial / \partial y)^{a},(\partial / \partial z)^{a}\right\}$ form an orthonormal basis, and $P_{i j}=\delta_{i}^{y} \delta_{j}^{y}+\delta_{i}^{z} \delta_{j}^{z}$. An index $\hat{t}$ will be used to denote contraction with the timelike normal $t^{a}$. We remark that the $\partial_{i} \psi_{a b}$ terms hidden in the omitted $\mathcal{C}_{i a b}$ terms in (14), (15) do not enter in the following because we already know that $\tilde{\psi}_{a b} \equiv 0$. 
The Fourier-Laplace transform of the constraint-preserving boundary conditions (26) then reads

$$
\begin{aligned}
\zeta \tilde{h}_{1}^{(\mathrm{C})} \doteq \tilde{c}_{\hat{t}}^{0-}= & \partial_{\xi}\left(-\frac{1}{2} \tilde{\Pi}_{\hat{t} \hat{t}}-\tilde{\Pi}_{x \hat{t}}-\frac{1}{2} \tilde{\Pi}_{x x}-\frac{1}{2} \tilde{\Pi}_{y y}-\frac{1}{2} \tilde{\Pi}_{z z}\right. \\
& \left.-\frac{1}{2} \tilde{\Phi}_{x \hat{t} \hat{t}}-\tilde{\Phi}_{x x \hat{t}}-\frac{1}{2} \tilde{\Phi}_{x x x}-\frac{1}{2} \tilde{\Phi}_{x y y}-\frac{1}{2} \tilde{\Phi}_{x z z}\right) \\
& +\mathrm{i}\left(-\tilde{\Pi}_{y \hat{t}}-\frac{1}{2} \tilde{\Phi}_{y \hat{t} \hat{t}}-\frac{1}{2} \tilde{\Phi}_{y x x}-\frac{1}{2} \tilde{\Phi}_{y y y}-\frac{1}{2} \tilde{\Phi}_{y z z}-\tilde{\Phi}_{x y \hat{t}}\right), \\
\zeta \tilde{h}_{2}^{(\mathrm{C})} \doteq \tilde{c}_{x}^{0-}= & \partial_{\xi}\left(-\frac{1}{2} \tilde{\Pi}_{\hat{t} \hat{t}}-\tilde{\Pi}_{\hat{t} x}-\frac{1}{2} \tilde{\Pi}_{x x}+\frac{1}{2} \tilde{\Pi}_{y y}+\frac{1}{2} \tilde{\Pi}_{z z}\right. \\
& \left.-\frac{1}{2} \tilde{\Phi}_{x \hat{t} \hat{t}}-\tilde{\Phi}_{x \hat{t} x}-\frac{1}{2} \tilde{\Phi}_{x x x}+\frac{1}{2} \tilde{\Phi}_{x y y}+\frac{1}{2} \tilde{\Phi}_{x z z}\right) \\
& +\mathrm{i}\left(-\tilde{\Pi}_{y x}-\tilde{\Phi}_{y \hat{t} x}-\tilde{\Phi}_{x y x}\right) \\
\zeta \tilde{h}_{3}^{(\mathrm{C})} \doteq \tilde{c}_{y}^{0-}= & \partial_{\xi}\left(-\tilde{\Pi}_{\hat{t} y}-\tilde{\Pi}_{x y}-\tilde{\Phi}_{x \hat{t} y}-\tilde{\Phi}_{x x y}\right) \\
& +\mathrm{i}\left(-\frac{1}{2} \tilde{\Pi}_{\hat{t} \hat{t}}+\frac{1}{2} \tilde{\Pi}_{x x}-\frac{1}{2} \tilde{\Pi}_{y y}+\frac{1}{2} \tilde{\Pi}_{z z}\right. \\
& \left.-\tilde{\Phi}_{y \hat{t} y}-\frac{1}{2} \tilde{\Phi}_{x y y}-\frac{1}{2} \tilde{\Phi}_{x \hat{t} \hat{t}}+\frac{1}{2} \tilde{\Phi}_{x x x}+\frac{1}{2} \tilde{\Phi}_{x z z}\right), \\
\zeta \tilde{h}_{4}^{(\mathrm{C})} \doteq \tilde{c}_{z}^{0-}= & \partial_{\xi}\left(-\tilde{\Pi}_{\hat{t} z}-\tilde{\Pi}_{x z}-\tilde{\Phi}_{x \hat{t} z}-\tilde{\Phi}_{x x z}\right)+\mathrm{i}\left(-\tilde{\Pi}_{y z}-\tilde{\Phi}_{y \hat{t} z}-\tilde{\Phi}_{x y z}\right) .
\end{aligned}
$$

$$
\begin{aligned}
& \zeta \tilde{h}_{5, a b}^{(\mathrm{C})} \doteq \tilde{c}_{x y a b}^{4}=\partial_{\xi} \tilde{\Phi}_{y a b}-\mathrm{i} \tilde{\Phi}_{x a b}, \\
& \zeta \tilde{h}_{6, a b}^{(\mathrm{C})} \doteq \tilde{c}_{x z a b}^{4}=\partial_{\xi} \tilde{\Phi}_{z a b} .
\end{aligned}
$$

Although it has to vanish for a solution that satisfies the constraints, we have added boundary data $\boldsymbol{h}^{(\mathrm{C})}$ to all the constraint-preserving boundary conditions in order to account for the inevitable numerical truncation error.

The physical boundary conditions (31) become

$$
\begin{aligned}
\zeta \tilde{h}_{1}^{(\mathrm{P})} \doteq \tilde{w}_{y y}^{-}= & \partial_{\xi}\left(-\frac{1}{2} \tilde{\Pi}_{y y}+\frac{1}{2} \Pi_{z z}-\frac{1}{2} \tilde{\Phi}_{x y y}+\frac{1}{2} \tilde{\Phi}_{x z z}\right) \\
& +\mathrm{i}\left(\frac{1}{2} \tilde{\Pi}_{y x}+\tilde{\Phi}_{x x y}+\tilde{\Phi}_{z z y}-\frac{1}{2} \tilde{\Phi}_{y x x}-\frac{1}{2} \tilde{\Phi}_{x y \hat{t}}+\frac{1}{2} \tilde{\Phi}_{y x \hat{t}}\right), \\
\zeta \tilde{h}_{2}^{(\mathrm{P})} \doteq \tilde{w}_{y z}^{-}= & \partial_{\xi}\left(-\tilde{\Pi}_{y z}-\tilde{\Phi}_{x y z}\right) \\
& +\mathrm{i}\left(\frac{1}{2} \tilde{\Pi}_{z x}+\tilde{\Phi}_{x x z}+\frac{1}{2} \tilde{\Phi}_{z z z}-\frac{1}{2} \tilde{\Phi}_{z x x}-\frac{1}{2} \tilde{\Phi}_{z y y}-\frac{1}{2} \tilde{\Phi}_{x z \hat{t}}+\frac{1}{2} \tilde{\Phi}_{z x \hat{t}}\right) .
\end{aligned}
$$

Note that the terms involving a normal derivative of $u_{a b}^{0} \equiv \psi_{a b}$ in (32) do not contribute because the Fourier-Laplace transform of $\psi_{a b}$ vanishes as a consequence of the evolution equations.

Finally, the gauge boundary conditions (36) are

$$
\begin{aligned}
& \tilde{h}_{1}^{(\mathrm{G})} \doteq \tilde{\Pi}_{\hat{t} \hat{t}}-\tilde{\Pi}_{x t}+\tilde{\Phi}_{x \hat{t} \hat{t}}-\tilde{\Phi}_{x x \hat{t}}, \\
& \tilde{h}_{2}^{(\mathrm{G})} \doteq \tilde{\Pi}_{\hat{t} x}-\tilde{\Pi}_{x x}+\tilde{\Phi}_{x \hat{t} x}-\tilde{\Phi}_{x x x}, \\
& \tilde{h}_{3}^{(\mathrm{G})} \doteq \tilde{\Pi}_{\hat{t} y}-\tilde{\Pi}_{x y}+\tilde{\Phi}_{x \hat{t} y}-\tilde{\Phi}_{x x y}, \\
& \tilde{h}_{4}^{(\mathrm{G})} \doteq \tilde{\Pi}_{\hat{t} z}-\tilde{\Pi}_{x z}+\tilde{\Phi}_{x \hat{t} z}-\tilde{\Phi}_{x x z} .
\end{aligned}
$$

The next step in the calculation is to insert the general $L^{2}$ solutions into the FourierLaplace transforms of the boundary conditions. We obtain a system of linear equations

$$
C(\zeta) \sigma=\tilde{\boldsymbol{h}}
$$


We need to evaluate the determinant of the matrix $C$. The resulting expressions are rather lengthy and some computer algebra is helpful here. A program (available from the author upon request) has been written in the computer algebra language REDUCE [37] in order to obtain the following results. We discuss the cases $\beta \geqslant 0$ and $\beta<0$ separately.

Suppose first that $\beta \geqslant 0$. In this case, the general solution is (72) and the boundary conditions are (75)-(78), (81)-(86). We find

$$
\operatorname{det} C=\frac{-\left[\zeta-(1+\beta) \lambda_{1}^{-}\right]^{16}}{8 \zeta^{6} K_{1}^{10}} .
$$

The function $\zeta \rightarrow \zeta-(1+\beta) \lambda_{1}^{-}$maps the right half of the complex plane to the right half of the complex plane minus a circle of radius $[(1+\beta) /(1-\beta)]^{1 / 2}$. Hence, $\operatorname{det} C$ has no zeros $\zeta$ with $\operatorname{Re}(\zeta)>0$ and is bounded away from zero even as $\operatorname{Re}(\zeta) \rightarrow 0$. In the limit $|\zeta| \rightarrow \infty$, we have $\left|\lambda_{1}^{-}\right| \sim|\zeta|,\left|K_{1}\right| \sim|\zeta|$ and hence $\operatorname{det} C$ is bounded away from zero too. We conclude that both the determinant condition and the Kreiss condition are satisfied.

Next, we consider the case $\beta<0$. Suppose first that $\zeta \neq-\beta$ so that the general decaying solution is (73). Plugging this into the boundary conditions (75)-(86) yields

$$
\operatorname{det} C=\frac{-\left[\zeta-(1+\beta) \lambda_{1}^{-}\right]^{16}\left(\zeta^{2}-\beta^{2}\right)^{10}}{8 \zeta^{16} \beta^{20} K_{1}^{10} K_{2}^{10}} .
$$

By the same argument as above, $\operatorname{det} C$ has no zeros $\zeta \neq-\beta$ with $\operatorname{Re}(\zeta) \geqslant 0$. In the degenerate case $\zeta=-\beta$, we have to use the solution (74) instead and find

$$
\operatorname{det} C=\frac{\left(K_{1}+K_{2}\right)^{10}}{8 \beta^{26} K_{1}^{10} K_{2}^{20}} \neq 0 \text {. }
$$

We conclude that for arbitrary shift at the boundary (with a subluminal normal component), our initial-boundary value problem satisfies the determinant condition and the Kreiss condition in the high-frequency limit. In particular, there are no strong instabilities.

It is easy to see that this result is unchanged if the physical boundary conditions (31) are replaced with the improved conditions (34) of [33]. In the frozen-coefficient approximation used in this section, they correspond to successive applications of the operator $\left(t^{a}+n^{a}\right) \partial_{a}=\hat{\partial}_{t}-(1+\beta) \partial_{x}$ to the boundary conditions (31). Each produces an additional factor of $\zeta-(1+\beta) \lambda_{1}^{-}$in the expressions for $\operatorname{det} C$, and as argued above, this factor is bounded away from zero.

Finally, we ask what happens if we replace the gauge boundary conditions (35) with the alternative conditions (37). In this case, we obtain

$$
\operatorname{det} C= \begin{cases}\frac{-\left[\zeta-(1+\beta) \lambda_{1}^{-}\right]^{8}\left(\zeta-\beta \lambda_{1}^{-}\right)^{8}}{8 \zeta^{6} K_{1}^{10}} & \text { if } \beta \geqslant 0, \\ \frac{-\left[\zeta-(1+\beta) \lambda_{1}^{-}\right]^{8}\left(\zeta-\beta \lambda_{1}^{-}\right)^{8}\left(\zeta^{2}-\beta^{2}\right)^{10}}{8 \zeta^{16} \beta^{20} K_{1}^{10} K_{2}^{10}} & \text { if } \beta<0,\end{cases}
$$

so that $\operatorname{det} C$ always has a zero

$$
\zeta=-\beta \text {. }
$$

Both the determinant condition and the Kreiss condition are satisfied if the shift points towards the interior at the boundary $(\beta>0)$. However, if the shift points towards the exterior $(\beta<0)$, the IBVP is ill posed. For tangential shift $(\beta=0)$, the determinant condition is satisfied (and hence there are no strong instabilities), but the Kreiss condition is violated $(\zeta=0$ is a generalized eigenvalue). What happens in this case will be investigated in the following section. 


\section{Weak instabilities}

As we have seen in the previous section, the determinant condition (53) is sufficient to detect strong instabilities of the form (43). The question remains whether a given IBVP admits ill-posed modes with 'milder' than exponential time dependence, the so-called weak instabilities. Examples of such instabilities with linear time dependence have been found in a formulation of Maxwell's equations in a gauge with vanishing electrostatic potential [24] and in an Einstein-Christoffel formulation of general relativity [4].

In this section, we develop a technique that can be used to systematically search for weak instabilities with polynomial time dependence. The criterion turns out to be closely related to the Kreiss condition. We then apply our method to the generalized harmonic system and show that it does not suffer from such instabilities. Furthermore, we show that if we use the alternative gauge boundary conditions (37) instead, a weak instability does exist, and we construct it explicitly.

\subsection{General theory}

We consider again the general IBVP (38)-(40). As before, we Fourier transform with respect to the coordinates $x^{A}$ tangential to the boundary. However, instead of assuming an exponential time dependence as in the Laplace transform (43), we now look for modes whose time dependence is given by a polynomial of order $p \geqslant 1$, i.e. we make the ansatz

$$
\boldsymbol{u}\left(t, x^{i}\right)=\exp \left(\mathrm{i} \omega \hat{\omega}_{A} x^{A}\right) \sum_{\nu=0}^{p} \tilde{\boldsymbol{u}}^{(v)}\left(\omega x^{1}\right) \frac{(\omega t)^{\nu}}{\nu !},
$$

where $\omega>0, \hat{\omega}_{A} \hat{\omega}^{A}=1$, and $\tilde{\boldsymbol{u}}^{(p)} \neq \mathbf{0}$. Substituting this into the evolution equations (38) and evaluating order by order in $t$, we find (setting $\xi \equiv \omega x^{1}$ )

$$
\begin{aligned}
& A^{1} \partial_{\xi} \tilde{\boldsymbol{u}}^{(p)}+\mathrm{i} \hat{\omega}_{A} A^{A} \tilde{\boldsymbol{u}}^{(p)}=0, \\
& A^{1} \partial_{\xi} \tilde{\boldsymbol{u}}^{(p-1)}+\mathrm{i} \hat{\omega}_{A} A^{A} \tilde{\boldsymbol{u}}^{(p-1)}=\tilde{\boldsymbol{u}}^{(p)}, \\
& A^{1} \partial_{\xi} \tilde{\boldsymbol{u}}^{(v)}+\mathrm{i} \hat{\omega}_{A} A^{A} \tilde{\boldsymbol{u}}^{(v)}=\tilde{\boldsymbol{u}}^{(v+1)}, \quad 0 \leqslant v \leqslant p-2 .
\end{aligned}
$$

Let us also expand the boundary data $\boldsymbol{h}$ in a similar fashion as in (93), up to order $p-1$ for a reason that will become clear shortly,

$$
\boldsymbol{h}\left(t, x^{A}\right)=\exp \left(\mathrm{i} \omega \hat{\omega}_{A} x^{A}\right) \sum_{\nu=0}^{p-1} \tilde{\boldsymbol{h}}^{(\nu)} \frac{(\omega t)^{\nu}}{\nu !} .
$$

The boundary conditions (40) then read

$$
\begin{aligned}
& S^{1} \partial_{\xi} \tilde{\boldsymbol{u}}^{(p)}+\mathrm{i} \hat{\omega}_{A} S^{A} \tilde{\boldsymbol{u}}^{(p)} \doteq 0, \\
& S^{1} \partial_{\xi} \tilde{\boldsymbol{u}}^{(p-1)}+\mathrm{i} \hat{\omega}_{A} S^{A} \tilde{\boldsymbol{u}}^{(p-1)} \doteq 0, \\
& S^{1} \partial_{\xi} \tilde{\boldsymbol{u}}^{(v)}+\mathrm{i} \hat{\omega}_{A} S^{A} \tilde{\boldsymbol{u}}^{(v)} \doteq \tilde{\boldsymbol{h}}^{(v+1)}, \quad 0 \leqslant v \leqslant p-2 .
\end{aligned}
$$

Suppose that a solution $\tilde{\boldsymbol{u}}=\left(\tilde{\boldsymbol{u}}^{(0)}, \tilde{\boldsymbol{u}}^{(1)}, \ldots, \tilde{\boldsymbol{u}}^{(p)}\right)$ satisfying equations (94)-(96) and (98)-(100) exists. Then the solution at times $t>0$ is of order $\mathrm{O}\left(\omega^{p}\right)$ whereas the initial data are $\mathrm{O}\left(\omega^{0}\right)$ and (the time integral of ) the boundary data are $\mathrm{O}\left(\omega^{p-1}\right)$. Hence, the solution cannot be estimated in terms of the initial and boundary data as $\omega \rightarrow \infty$ and the IBVP is ill posed. We may actually argue that it is already ill posed if a (non-zero) solution of (94)-(96) 
exists that obeys the top two boundary conditions (98)-(99), for we can always choose the boundary data $\tilde{\boldsymbol{h}}$ such that the remaining boundary conditions (100) are satisfied.

Let us look more closely at equations (94) and (98); these are identical with equations (44) and (50) in section 3.1 for $s=0$. We conclude that if a weak instability exists, $s=0$ is a generalized eigenvalue. (Strictly speaking, one has to consider the Fourier-Laplace solutions for $\operatorname{Re}(s)>0$ and take the limit $s \rightarrow 0$. We assume here that the solutions are continuous at $s=0$, which is the case in all the examples we discuss.) Therefore, if the Kreiss condition is satisfied, there are no weak instabilities of any polynomial order $p$.

On the other hand, $s=0$ being a generalized eigenvalue does not automatically imply that there is a weak instability. One still has to solve the coupled ODE system (94)-(96) and impose the boundary conditions (98)-(99) on its solution.

In [24] it is claimed that (for a certain choice of parameters) the Maxwell system considered there admits a weak instability (with $p=1$ ) even though the Kreiss condition is satisfied. This is obviously a contradiction to the result that we have just derived. However, the eigenvectors $\boldsymbol{w}_{j}$ appearing in the general $L^{2}$ solution in [24, equation (51)] are normalized in such a way that they become infinite as $s \rightarrow 0$, and the estimate (54) does not obtain. If the correct normalization is used, one finds that $s=0$ is indeed a generalized eigenvalue.

\subsection{Application to the GH system}

To see how the method outlined above works in practice, we apply it to the generalized harmonic Einstein equations in the high-frequency limit (equations (60)-(62)).

First, we take the gauge boundary conditions to be (35). We have already seen in section 3.2 that the Kreiss condition is satisfied, and hence there are no weak instabilities with polynomial time dependence. (One can also carry out the explicit calculation below, obtaining the same result.)

Next, we consider the alternative gauge boundary conditions (37), and we take the shift to be tangential at the boundary $(\beta=0)$. As discussed in section 3.2, the Kreiss condition is violated in this case, with $s=0$ being a generalized eigenvalue. This indicates that there might be a weak instability. Let us search for one with $p=1$ and homogeneous boundary data. Equation (94) implies

$$
\begin{aligned}
& -\partial_{\xi} \tilde{\Phi}_{x a b}^{(1)}-\mathrm{i} \tilde{\Phi}_{y a b}^{(1)}=0, \\
& -\mathrm{i} \tilde{\Pi}_{a b}^{(1)}=0,
\end{aligned}
$$

and (95) reads

$$
\begin{aligned}
& -\partial_{\xi} \tilde{\Phi}_{x a b}^{(0)}-\mathrm{i} \tilde{\Phi}_{y a b}^{(0)}=\tilde{\Pi}_{a b}^{(1)}, \\
& -\partial_{\xi} \tilde{\Pi}_{a b}^{(0)}=\tilde{\Phi}_{x a b}^{(1)}, \\
& -\mathrm{i} \Pi_{a b}^{(0)}=\tilde{\Phi}_{y a b}^{(1)}, \\
& 0=\tilde{\Phi}_{z a b}^{(1)} .
\end{aligned}
$$

Substituting (104), (105) into (101), we obtain

$$
\partial_{\xi}^{2} \tilde{\Pi}_{a b}^{(0)}=\tilde{\Pi}_{a b}^{(0)} \Rightarrow \tilde{\Pi}_{a b}^{(0)}=\sigma_{1 a b} \mathrm{e}^{-\xi} .
$$

Assuming that $\mathcal{C}_{i a b}=0$ initially, i.e.

$$
\tilde{\Phi}_{x a b}^{(0)}=\omega \partial_{\xi} \tilde{\psi}_{a b}^{(0)}, \quad \tilde{\Phi}_{y a b}^{(0)}=\mathrm{i} \omega \tilde{\psi}_{a b}^{(0)}, \quad \tilde{\Phi}_{z a b}^{(0)}=0,
$$


equation (103) together with (102) similarly implies

$$
\partial_{\xi}^{2} \tilde{\psi}_{a b}^{(0)}=\tilde{\psi}_{a b}^{(0)} \Rightarrow \tilde{\psi}_{a b}^{(0)} \equiv \omega^{-1} \sigma_{2 a b} \mathrm{e}^{-\xi} .
$$

Hence, the most general $L^{2}$ solution (consistent with $\mathcal{C}_{i a b}=0$ ) is of the form

$$
\begin{aligned}
& \left(\tilde{\Pi}_{a b}^{(0)}, \tilde{\Phi}_{x a b}^{(0)}, \tilde{\Phi}_{y a b}^{(0)}, \tilde{\Phi}_{z a b}^{(0)}\right)=\mathrm{e}^{-\xi}\left(\sigma_{1 a b},-\sigma_{2 a b}, \mathrm{i} \sigma_{2 a b}, 0\right), \\
& \left(\tilde{\Pi}_{a b}^{(1)}, \tilde{\Phi}_{x a b}^{(1)}, \tilde{\Phi}_{y a b}^{(1)}, \tilde{\Phi}_{z a b}^{(1)}\right)=\mathrm{e}^{-\xi}\left(0, \sigma_{1 a b},-\mathrm{i} \sigma_{1 a b}, 0\right) .
\end{aligned}
$$

Only the mode associated with $\sigma_{1 a b}$ corresponds to a solution with $\tilde{\boldsymbol{u}}^{(1)} \neq \mathbf{0}$. Substituting this mode into the Fourier transform of the homogeneous boundary conditions and evaluating order by order in $t$, one obtains a $20 \times 10$ linear system of equations for the constants $\sigma_{1 a b}$. The only non-trivial solution is given by

$$
\sigma_{1 x x}=1, \quad \sigma_{1 x y}=-\mathrm{i}, \quad \sigma_{1 y y}=-1, \quad \text { all other } \quad \sigma_{k a b}=0 .
$$

It corresponds to setting

$$
\begin{array}{ll}
\Pi_{i j}=f_{, i j}, & \Pi_{0 a}=0, \\
\Phi_{k i j}=-t f_{, k i j}, & \Phi_{k 0 a}=0, \\
f \equiv \mathrm{e}^{\omega(-x+\mathrm{i} y)} &
\end{array}
$$

which is of the same type as the weak instabilities found in [4, 24].

A similar calculation shows that there are no weak instabilities of order $p \geqslant 2$ for homogeneous boundary data. However, as explained in section 4.1, this is not true for inhomogeneous boundary data.

\section{Robust stability tests}

In this section, we perform a series of robust stability tests on our numerical implementation of the GH system. This test involves adding small random perturbations to a known exact solution. Thus, all possible frequencies at a given resolution are excited. By increasing the resolution and looking at the growth rate of the numerical solution, this test is very effective in spotting ill-posed modes. The robust stability test was suggested in the context of the Cauchy problem by the 'Apples with Apples' collaboration ([38], see also [39] for an application to the spectral evolution code used for this work). Previous studies in which a version of the test was used in relation to the stability of the IBVP include $[4,14,17,18,40]$.

For most of the tests, the background solution is taken to be flat space with a constant shift $N^{i}$,

$$
\mathrm{d} s^{2}=-\mathrm{d} t^{2}+\delta_{i j}\left(\mathrm{~d} x^{i}+N^{i} \mathrm{~d} t\right)\left(\mathrm{d} x^{j}+N^{j} \mathrm{~d} t\right) .
$$

We remark that this is the most general background solution in the high-frequency limit, as discussed in section 3.2. The spatial domain is taken to be of topology $T^{2} \times \mathbb{R}$. This domain is intended to be as simple as possible, avoiding additional complications due to sharp corners and edges. We take $x, y, z \in[-0.5,0.5]$. The boundary conditions discussed in this paper are imposed at $x= \pm 0.5$, and periodic boundary conditions are imposed in the $y$ and $z$ directions.

For the last test, we consider instead the Schwarzschild solution in Kerr-Schild coordinates,

$\mathrm{d} s^{2}=-\left(1-\frac{2 M}{r}\right) \mathrm{d} t^{2}+\frac{4 M}{r} \mathrm{~d} r \mathrm{~d} t+\left(1+\frac{2 M}{r}\right) \mathrm{d} r^{2}+r^{2}\left(\mathrm{~d} \theta^{2}+\sin ^{2} \theta \mathrm{d} \phi^{2}\right)$. 
In this case, the spatial domain is taken to be a spherical shell extending from $r_{\text {min }}=1.8 M$ (just inside the event horizon) to $r_{\max }=11.8 \mathrm{M}$. The boundary conditions discussed in this paper are imposed at the outer boundary $r=r_{\max }$ (where the shift is outward-pointing, the $\beta<0$ case in section 3.2). No boundary conditions are imposed at the inner boundary $r=r_{\min }$. See also [41] for a spherically symmetric robust stability test on Schwarzschild spacetime in Painlevé-Gullstrand coordinates.

As initial data, we compute the variables $\psi_{a b}, \Pi_{a b}$ and $\Phi_{i a b}$ corresponding to the exact solutions (114), (115) and add random noise of amplitude $\epsilon=10^{-10}$ (in the flat-space case) or $\epsilon=10^{-6}$ (in the Schwarzschild case) to them. In addition, random noise of the same amplitude is added both to the right-hand sides of the expressions for the time derivatives at the boundary (118) and to the right-hand sides of the evolution equations. In this way, we probe the influence of non-zero initial and source data as well, which could not be analysed using the methods of section 3.

For more physically realistic tests involving black hole spacetimes, we refer the reader to [27]. In particular, an ingoing gravitational wave was injected through the outer boundary (i.e. data $h^{(\mathrm{P})}$ were prescribed in equation $\left.(31)\right)$. The gravitational radiation scattered back by the black hole was extracted, correctly reproducing the dominant quasi-normal mode oscillation.

\subsection{Numerical implementation}

We use pseudospectral methods as described, for example, in [3].

For the flat-space tests on $T^{2} \times \mathbb{R}$, the numerical solution is expanded into Chebyshev polynomials in $x$ and into Fourier series in $y$ and $z$. We use between 9 and 27 basis functions in the $x$ and $y$ directions (these are typical resolutions in realistic numerical relativity simulations using spectral methods) and 3 basis functions in the $z$ direction. Reducing the effective dimension by one enables us to evolve up to final times of $t=1000$ within a tolerable runtime of the code even for the higher resolutions. We have checked for moderate resolutions that the behaviour is qualitatively unchanged in fully three-dimensional runs. A fourth-order RungeKutta scheme is used for the time integration, with a Courant factor of $\Delta t / \Delta x_{\min }=1.5$, where $x_{\min }$ is the minimum spacing between the (in the Chebyshev case, non-uniform) pseudospectral collocation points.

For the Schwarzschild test, the numerical solution is expanded into Chebyshev polynomials in $r$ and spherical harmonics in the angular directions. Here, we use between 15 and 27 radial and between 11 and 19 angular basis functions. The same time integration scheme with the same Courant factor is used as in the flat-space case. The highest-order spherical harmonics are filtered as described, for example, in [3]. No filtering is applied to the Chebyshev and Fourier expansions.

The boundary conditions are implemented by prescribing the time derivatives of the characteristic variables at the boundary [42]. For the Neumann-like constraint-preserving boundary conditions (26), (29) and physical boundary conditions (31), we use the evolution equations for the incoming characteristic variables in order to treat normal derivatives for time derivatives,

$$
\begin{aligned}
& d_{t} u_{a b}^{1-}=\left(N^{n}+N\right) d_{n} u_{a b}^{1-}+\text { tangential derivatives } \\
& d_{t} u_{A a b}^{2}=N^{n} d_{n} u_{A a b}^{2}+\text { tangential derivatives. }
\end{aligned}
$$

(The notation $d$ instead of $\partial$ indicates that the partial derivative acts only on the fundamental variables, not on the eigenvectors needed to form the characteristic variables [27].) For the gauge boundary conditions, we simply take a time derivative of (35). In this way, we obtain 

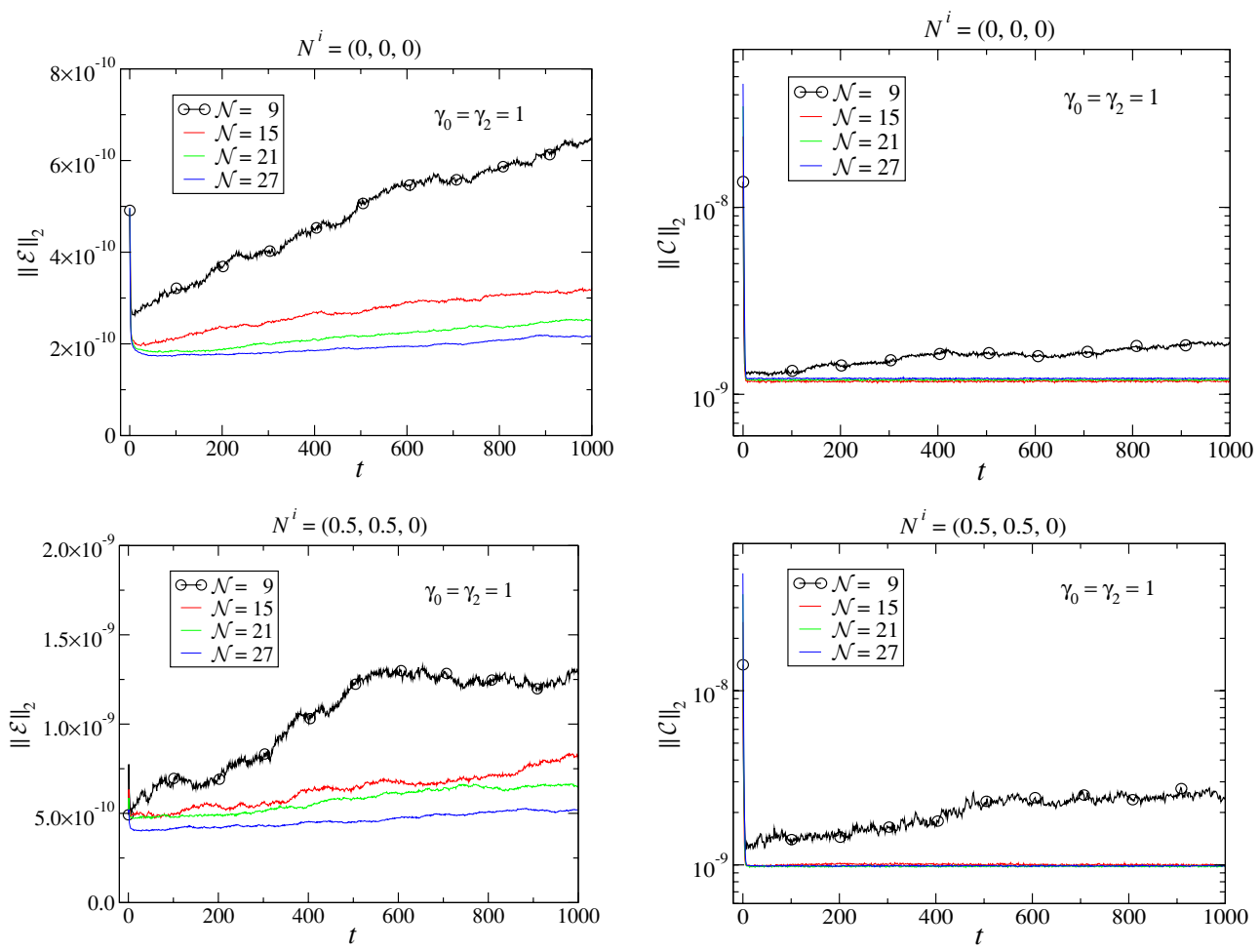

Figure 1. Robust stability test on the flat-space background (114) with constraint damping. $L^{2}$ norms of the error (left) and the constraints (right) for two different shift vectors $N^{i}$ and for four different resolutions $\mathcal{N} \equiv \mathcal{N}_{x}=\mathcal{N}_{y}$.

expressions for $d_{t} u^{2}, P^{(\mathrm{C})} d_{t} u^{1-}, P^{(\mathrm{P})} d_{t} u^{1-}$ and $P^{(\mathrm{G})} d_{t} u^{1-}$ (omitting the spacetime indices for simplicity). These are then combined to form

$$
d_{t} u^{1-} \doteq P^{(\mathrm{C})} d_{t} u^{1-}+P^{(\mathrm{P})} d_{t} u^{1-}+P^{(\mathrm{G})} d_{t} u^{1-}
$$

at the boundary (note that $P^{(\mathrm{C})}, P^{(\mathrm{P})}$ and $P^{(\mathrm{G})}$ are mutually orthogonal projection operators adding up to the identity). In order to implement the alternative gauge boundary conditions (37), we first fill $P^{(\mathrm{C})} d_{t} u^{1-}$ and $P^{(\mathrm{P})} d_{t} u^{1-}$ as before and then set

$$
P^{(\mathrm{G})} d_{t} u^{1-} \doteq Q P^{(\mathrm{C})} d_{t} u^{1-}+\left(Q-P^{(\mathrm{G})}\right) d_{t}\left(u^{1+}+2 \gamma_{2} \psi\right),
$$

where the operator $Q$ has the properties $P^{(\mathrm{C})} Q=P^{(\mathrm{P})} Q=0, P^{(\mathrm{G})} Q=Q, Q P^{(\mathrm{C})}=Q$, $Q P^{(\mathrm{P})}=Q P^{(\mathrm{G})}=0$ and $t \cdot\left(P^{(\mathrm{C})}+Q\right)=0$. It then follows from (118) and (10) that $d_{t}(t \cdot \Pi) \doteq 0$ at the boundary, as desired. An explicit expression for $Q$ is given by

$$
Q_{a b}{ }^{c d}=k_{a} k_{b} k^{c} k^{d}+2 k_{(a} P_{b)}{ }^{(c} k^{d)}-2 k_{(a} l_{b)} k^{c} k^{d} .
$$

\subsection{Numerical results}

The following plots show the $L^{2}$ norms of the error

$$
\mathcal{E}=\sqrt{\left(\delta \psi_{a b}\right)^{2}+\left(\delta \Pi_{a b}\right)^{2}+\left(\delta \Phi_{i a b}\right)^{2}}
$$



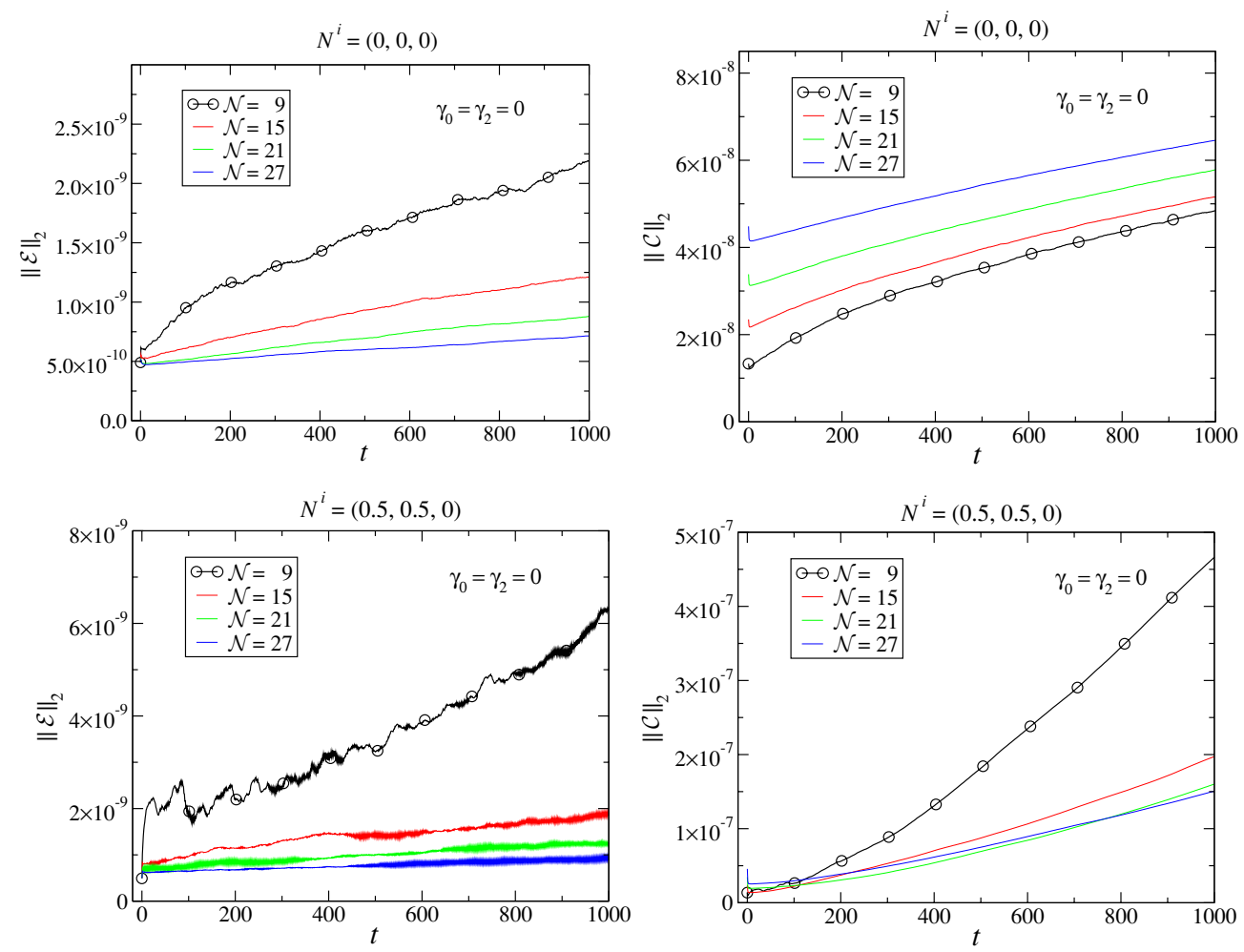

Figure 2. Robust stability test on the flat-space background (114) without constraint damping. $L^{2}$ norms of the error (left) and the constraints (right) for two different shift vectors $N^{i}$ and for four different resolutions $\mathcal{N} \equiv \mathcal{N}_{x}=\mathcal{N}_{y}$.

and of the constraints

$$
\mathcal{C}=\sqrt{\left(C_{a}\right)^{2}+\left(\mathcal{F}_{a}\right)^{2}+\left(\mathcal{C}_{i a}\right)^{2}+\left(\mathcal{C}_{i a b}\right)^{2}+\left(\mathcal{C}_{i j a b}\right)^{2}}
$$

as functions of time. In the above, the notation $\left(M_{a b \cdots c}\right)^{2}$ refers to the sum of the squares of the Cartesian components of $M_{a b \cdots c}$. The differences $\delta$ in (121) are taken with respect to the background solutions (114), (115). When computing the quantity $\delta \psi_{a b}$, we subtract its spatial average from it. This is because the constant mode of $\psi_{a b}$ typically has a large linear drift in time caused by a non-zero constant mode of $\Pi_{a b}$ (which is not eliminated by the differential boundary conditions). Note that this procedure does not affect the higher-frequency modes.

We begin with the flat-space background (114). We run the test for two different shift vectors, $N^{i}=(0,0,0)$ and $N^{i}=(0.5,0.5,0)$. In the latter case, the shift points towards the interior at the $x=-0.5$ boundary (corresponding to the $\beta>0$ case in section 3.2) and towards the exterior at the $x=0.5$ boundary (corresponding to the $\beta<0$ case), and the shift also has a component tangential to the boundary. Figure 1 shows that in both cases, the error and the constraints remain of the same order as initially up to $t=1000$ (and presumably forever). For this run, we included constraint damping with parameters $\gamma_{0}=\gamma_{2}=1$ (the same choice was made in [27] in order to obtain long-term stable black hole evolutions). This leads to the sharp initial decrease of the constraint violations (note that the randomly perturbed initial data do not satisfy the constraints). Because one might suspect that the constraint damping might 

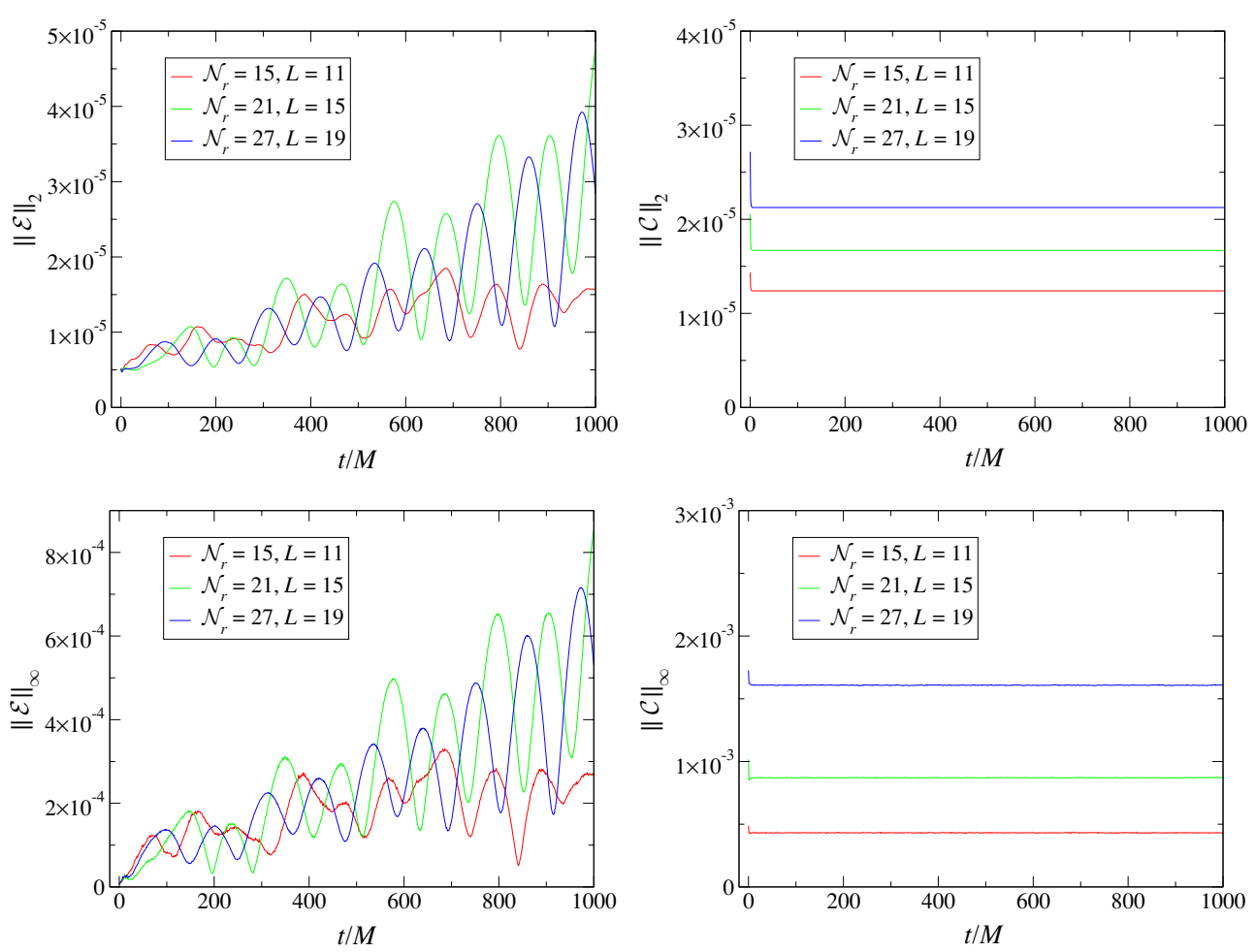

Figure 3. Robust stability test on the Schwarzschild background (115). $L^{2}$ (top) and $L^{\infty}$ (bottom) norms of the error (left) and the constraints (right) for three different resolutions.

somehow hide potential instabilities, we rerun the test without constraint damping (figure 2). Now the constraints grow slightly (as expected), but still there is no sign of an instability.

Next, we turn to the Schwarzschild background (115). Here, we choose the amplitude of the random perturbations somewhat higher than in the flat-space case $\left(10^{-6}\right.$ rather than $10^{-10}$ ) so that it is much larger than the error incurred during an evolution of the unperturbed Schwarzschild spacetime for the resolutions considered. In this case, the constraint damping is essential in order to avoid exponential growth of the constraints [27]. Figure 3 shows the results of this test, using both the $L^{2}$ norm and the $L^{\infty}$ norm (which is more sensitive to local effects on the boundary). There are now significant oscillations in the error due to the much higher amplitude of the perturbations, but they grow only linearly on an average and at a rate that does not appear to increase significantly with resolution. The constraints remain essentially constant, thanks to the constraint damping. We remark that as a consequence of the stability analysis in section 3.2, flat space and Schwarzschild spacetime should be equivalent with regard to stability in the high-frequency limit.

The numerical results strongly suggest that the initial-boundary value problem is indeed well posed, even if non-trivial initial and source data are included. Previous studies [4, 14, $17,18,40]$ presented similar numerical evidence (for different formulations), although only initial and boundary data but not source data were included in those robust stability tests.

Finally, we return to flat space (114), but now we replace the gauge boundary conditions (35) with the alternative set (37). The top half of figure 4 shows the results of the robust stability test for shift vector $N^{i}=(0.5,0,0)$. Now the error grows exponentially at a rate that 

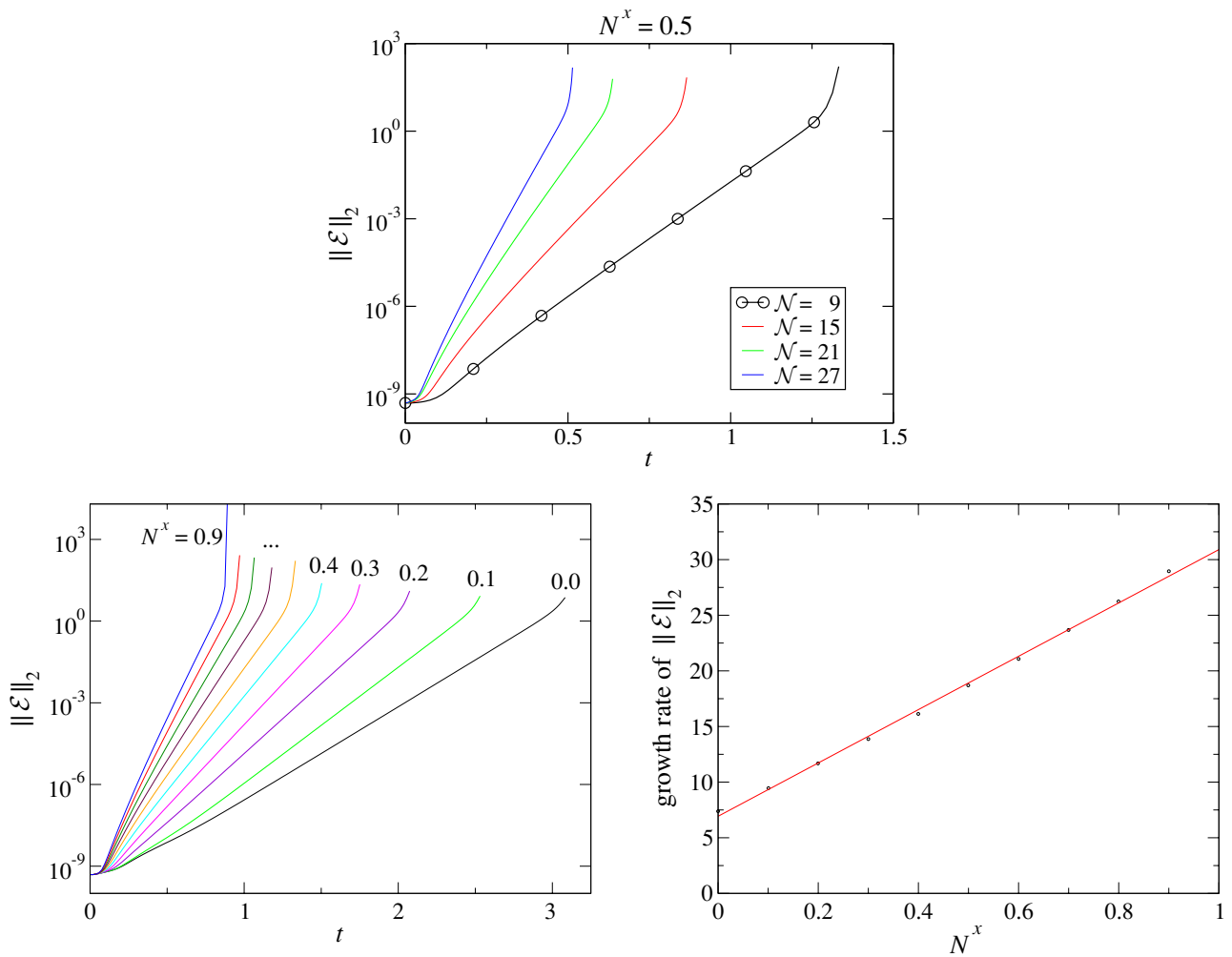

Figure 4. Robust stability test on the flat-space background (114), using the alternative gauge boundary conditions (37). Top half: $L^{2}$ norm of the error for four different resolutions $\mathcal{N} \equiv \mathcal{N}_{x}=\mathcal{N}_{y}$, using $N^{x}=0.5$ for the normal component of the shift. Bottom half: $L^{2}$ norm of the error for various values of $N^{x}$ at fixed resolution $\mathcal{N}=9$, and linear fit to the growth rate. (In all cases, $N^{y}=N^{z}=0$ and $\gamma_{0}=\gamma_{2}=1$.)

increases with resolution (note also the timescale as compared with figures 1-3). This is what we expect because the initial-boundary value problem is ill posed in this case (section 3.2). The bottom half of figure 4 shows what happens if we vary the normal component $N^{x}$ of the shift. As predicted by the Fourier-Laplace analysis in section 3.2 (cf equation (92)), the growth rate depends linearly on $N^{x}$. Numerically, we find a non-zero offset of the growth rate at $N^{x}=0$, which is not obvious from the analysis. We remark however that because of the random perturbations, $N^{x}$ will never exactly vanish at the boundary, and furthermore the argument in section 4 indicates that even for $N^{x}=0$, there are polynomial instabilities of an arbitrarily high polynomial order if non-zero boundary data are included.

\section{Conclusions}

We considered the initial-boundary value problem for a first-order formulation of the Einstein equations in generalized harmonic gauge. This system was derived in [27] and has proven very successful in obtaining long-term stable black hole evolutions. The boundary conditions we considered have the special property that they control the incoming gravitational radiation via 
the incoming fields of the Weyl tensor [1-4]. We believe that this is essential in order to obtain reliable information about the gravitational radiation emitted from a compact source if the domain of integration has artificial timelike boundaries, as is a common situation in numerical relativity. In addition, the boundary conditions eliminate the incoming constraint fields $[3,5$, $10,11,14]$, in which we believe they are superior to constraint-preserving boundary conditions of the Dirichlet type [20] from a numerical point of view.

In section 3, we analysed the well posedness of the initial-boundary value problem using the Fourier-Laplace technique [4, 5, 20, 23-25]. This required taking the high-frequency, or frozen-coefficient approximation. To allow for an arbitrary background spacetime, we had to take into account an arbitrary shift vector at the boundary. This generalizes the result stated in [27], where only a tangential shift was considered. We showed that the Kreiss condition is satisfied, which implies that the system is boundary stable (i.e. the solution can be estimated in terms of the boundary data). Unlike for maximally dissipative boundary conditions, it is not known in the present case of differential boundary conditions whether (or under which additional assumptions) the Kreiss condition is also sufficient for well posedness if non-trivial initial and source data are included. It would be of considerable interest to the numerical relativity community to obtain a general theorem covering this case.

It has been claimed $[4,24]$ that systems with differential boundary conditions might admit weak instabilities with milder than exponential time dependence even if the Kreiss condition is satisfied. In section 4 , we considered instabilities with polynomial time dependence for a general first-order initial-boundary value problem. It was found that such instabilities are ruled out by the Kreiss condition. (More precisely, the condition is that 0 be not a generalized eigenvalue.) For the generalized harmonic Einstein equations, it turned out that the choice of boundary conditions for the gauge degrees of freedom can be crucial for stability; for an innocent-looking set of alternative gauge boundary conditions that did not satisfy the Kreiss condition, we found a weak instability (with linear time dependence) if the shift was tangential at the boundary. However, as soon as the shift pointed towards the exterior, the weak instability was turned into a strong one with exponential time dependence-this demonstrates that taking into account a normal component of the shift can be important.

Finally, we performed a numerical robust stability test $[4,14,17,18,38-41]$. The background spacetime was taken to be either Minkowski space with a shift or Schwarzschild. We added small random perturbations to both the initial data, the boundary conditions and the right-hand side of the evolution equations. The generalized harmonic evolution system performed very well on these tests, with the error and the constraints remaining of the same order of magnitude over 1000 light crossing times in the flat-space case, or $1000 \mathrm{M}$ in the Schwarzschild case. This strongly suggests that the initial-boundary value problem is likely to be well posed even if non-trivial initial and source data are included. We also ran the test on the alternative set of gauge boundary conditions, finding resolution-dependent exponential growth of the error as predicted by the Fourier-Laplace analysis. The linear dependence of the growth rate on the normal component of the shift was also reproduced, although exponential growth was observed even in the limiting case of a tangential shift.

\section{Acknowledgments}

I am particularly grateful to Olivier Sarbach for many valuable discussions and to Mark Scheel for help with the Caltech-Cornell Spectral Einstein Code, which he developed jointly with Larry Kidder and Harald Pfeiffer. I further thank Lee Lindblom for suggesting this project and for encouragement throughout the work, Alexander Alekseenko for helpful discussions, and all of them for careful reading of the manuscript. This work was supported in part by a grant 
from the Sherman Fairchild Foundation, by NSF grants PHY-0244906 and PHY-0601459 and by NASA grants NAG5-12834 and NNG05GG52G.

\section{Appendix}

In this appendix, we prove a detail that we postponed in section 2.2. Recall that in the case of an outward-pointing shift $\left(N^{x}<0\right)$, we could only impose constraint-preserving boundary conditions on the incoming constraint fields $c_{a}^{0-}$ and $c_{n A a b}^{4}$. Here, we show that this implies that the remaining incoming constraint fields $c_{A a}^{2}$ and $c_{A B a b}^{4}$ also vanish at the boundary so that we have a full set of maximally dissipative boundary conditions for the constraint evolution system. We restrict ourselves to the high-frequency limit and use the Fourier-Laplace framework of section 3.2.

Suppose that we impose the constraint-preserving boundary conditions proposed in section 2.2 in the $N^{x}<0$ case: in Fourier-Laplace language, equations (75)-(86) with zero data $\tilde{h}^{\mathrm{C}}$. These imply a linear system

$$
C^{(1)} \boldsymbol{\sigma}=0
$$

for the constants $\sigma_{i a b}$ parametrizing the general Fourier-Laplace-transformed solutions (73). The kernel of $C^{(1)}$ spans all solutions that satisfy our constraint-preserving boundary conditions.

Next, we consider the remaining incoming constraints $c_{A a}^{2}$ and $c_{A B a b}^{4}$. Their FourierLaplace transforms are given by

$$
\begin{aligned}
& \tilde{c}_{y \hat{t}}^{2}=\partial_{\xi} \tilde{\Phi}_{y x \hat{t}}+\mathrm{i}\left(\tilde{\Phi}_{y y \hat{t}}+\frac{1}{2} \tilde{\Pi}_{\hat{t} \hat{t}}+\frac{1}{2} \tilde{\Pi}_{x x}+\frac{1}{2} \tilde{\Pi}_{y y}+\frac{1}{2} \tilde{\Pi}_{z z}\right), \\
& \tilde{c}_{y x}^{2}=\partial_{\xi}\left(\frac{1}{2} \tilde{\Phi}_{y x x}+\frac{1}{2} \tilde{\Phi}_{y \hat{t} \hat{t}}-\frac{1}{2} \tilde{\Phi}_{y y y}-\frac{1}{2} \tilde{\Phi}_{y z z}\right)+\mathrm{i}\left(\tilde{\Phi}_{y y x}+\tilde{\Pi}_{\hat{t} x}\right), \\
& \tilde{c}_{y y}^{2}=\partial_{\xi} \tilde{\Phi}_{y x y}+\mathrm{i}\left(\frac{1}{2} \tilde{\Phi}_{y y y}+\frac{1}{2} \tilde{\Phi}_{y \hat{t} \hat{t}}-\frac{1}{2} \tilde{\Phi}_{y x x}-\frac{1}{2} \tilde{\Phi}_{y z z}+\tilde{\Pi}_{\hat{t} y}\right), \\
& \tilde{c}_{y z}^{2}=\partial_{\xi} \tilde{\Phi}_{y x z}+\mathrm{i}\left(\tilde{\Phi}_{y y z}+\tilde{\Pi}_{\hat{t} z}\right) \\
& \tilde{c}_{z \hat{t}}^{2}=\partial_{\xi} \tilde{\Phi}_{z x \hat{t}}+\mathrm{i} \tilde{\Phi}_{z y \hat{t}}, \\
& \tilde{c}_{z x}^{2}=\partial_{\xi}\left(\frac{1}{2} \tilde{\Phi}_{z x x}+\frac{1}{2} \tilde{\Phi}_{z \hat{t} \hat{t}}-\frac{1}{2} \tilde{\Phi}_{z y y}-\frac{1}{2} \tilde{\Phi}_{z z z}\right)+\mathrm{i} \tilde{\Phi}_{z y x}, \\
& \tilde{c}_{z y}^{2}=\partial_{\xi} \tilde{\Phi}_{z x y}+\mathrm{i}\left(\frac{1}{2} \tilde{\Phi}_{z y y}+\frac{1}{2} \tilde{\Phi}_{z \hat{t} \hat{t}}-\frac{1}{2} \tilde{\Phi}_{z x x}-\frac{1}{2} \tilde{\Phi}_{z z z}\right), \\
& \tilde{c}_{z z}^{2}=\partial_{\xi} \tilde{\Phi}_{z x z}+\mathrm{i} \tilde{\Phi}_{z y z}, \\
& \tilde{c}_{y z a b}^{4}=\mathrm{i} \tilde{\Phi}_{z a b} .
\end{aligned}
$$

Equations (A.2)-(A.10) imply another linear system for the integration constants $\sigma_{i a b}$,

$$
C^{(2)} \boldsymbol{\sigma}=0 .
$$

We need to check that any solution satisfying the boundary conditions (75)-(86) also satisfies equations (A.2)-(A.10), in other words, that

$$
\operatorname{ker} C^{(1)} \subset \operatorname{ker} C^{(2)} \text {. }
$$

This is a purely algebraic condition, which is straightforward to check with our computer algebra program. It is indeed satisfied. 


\section{References}

[1] Friedrich H and Nagy G 1999 The initial boundary value problem for Einstein's vacuum field equations Commun. Math. Phys. 201 619-55

[2] Bardeen J M and Buchman L T 2002 Numerical tests of evolution systems, gauge conditions, and boundary conditions for 1D colliding gravitational plane waves Phys. Rev. D 65064037

[3] Kidder L E, Lindblom L, Scheel M A, Buchman L T and Pfeiffer H P 2005 Boundary conditions for the Einstein evolution system Phys. Rev. D 71064020

[4] Sarbach O and Tiglio M 2005 Boundary conditions for Einstein's field equations: analytical and numerical analysis J. Hyp. Diff. Eq. 2 839-83

[5] Stewart J M 1998 The Cauchy problem and the initial boundary value problem in numerical relativity Class. Quantum Grav. 15 2865-89

[6] Rauch J 1985 Symmetric positive systems with boundary characteristics of constant multiplicity Trans. Am. Math. Soc. 291 167-87

[7] Secchi P 1996 The initial boundary value problem for linear symmetric hyperbolic systems with characteristic boundary of constant multiplicity Diff. Int. Eq. 9 671-700

[8] Secchi P 1996 Well-posedness of characteristic symmetric hyperbolic systems Arch. Ration. Mech. Anal. 134 155-97

[9] Buchman L T and Bardeen J M 2005 Schwarzschild tests of the Wahlquist-Estrabrook-Buchman-Bardeen tetrad formulation for numerical relativity Phys. Rev. D 72124014

[10] Iriondo M S and Reula O A 2002 Free evolution of self-gravitating, spherically symmetric waves Phys. Rev. D 65044024

[11] Calabrese G, Lehner L and Tiglio M 2002 Constraint-preserving boundary conditions in numerical relativity Phys. Rev. D 65104031

[12] Calabrese G, Pullin J, Reula O, Sarbach O and Tiglio M 2003 Well posed constraint-preserving boundary conditions for the linearized Einstein equations Commun. Math. Phys. 240 377-95

[13] Gundlach C and Martín-García J M 2004 Symmetric hyperbolicity and consistent boundary conditions for second-order Einstein equations Phys. Rev. D 70044032

[14] Bona C, Ledvinka T, Palenzuela-Luque C and Žáček M 2005 Constraint-preserving boundary conditions in the Z4 numerical relatiity formalism Class. Quantum Grav. 22 2615-34

[15] Tarfulea N 2005 Constraint-preserving boundary conditions for hyperbolic formulations of Einstein's equations PhD Thesis University of Minnesota Preprint gr-qc/0508014

[16] Alekseenko A 2004 Constraint-preserving boundary conditions for the linearized BSSN formulation Preprint gr-qc/0405080

[17] Szilágyi B, Schmidt B G and Winicour J 2002 Boundary conditions in linearized harmonic gravity Phys. Rev. D 65064015

[18] Szilágyi B and Winicour J 2003 Well-posed initial-boundary evolution in general relativity Phys. Rev. D 68041501

[19] Babiuc M C, Szilágyi B and Winicour J 2006 Harmonic initial-boundary evolution in general relativity Phys. Rev. D 73064017

[20] Kreiss H O and Winicour J 2006 Problems which are well-posed in a generalized sense with applications to the Einstein equations Class. Quantum Grav. 16 S405-20

[21] Kreiss H O, Gustafsson B and Oliger J 1995 Time Dependent Problems and Difference Methods (New York: Wiley)

[22] Kreiss H O and Lorenz J 1989 Initial-Boundary Value Problems and the Navier-Stokes Equations (New York: Academic)

[23] Calabrese G and Sarbach O 2003 Detecting ill-posed boundary conditions in general relativity J. Math. Phys. 44 3888-99

[24] Reula O and Sarbach O 2005 A model problem for the initial-boundary value problem of Einstein's field equations J. Hyp. Diff. Eq. 2 397-435

[25] Rinne O 2005 Axisymmetric numerical relativity PhD Thesis University of Cambridge Preprint gr-qc/0601064

[26] Nagy G and Sarbach O 2006 A minimization problem for the lapse and the initial-boundary value problem for Einstein's field equations Class. Quantum Grav. 16 S477-504

[27] Lindblom L, Scheel M A, Kidder L E, Owen R and Rinne O 2006 A new generalized harmonic evolution system Class. Quantum Grav. 16 S447-62

[28] Pretorius F 2005 Evolution of binary black hole spacetimes Phys. Rev. Lett. 95121101

[29] Pretorius F 2006 Simulation of binary black hole spacetimes with a harmonic evolution scheme Class. Quantum Grav. 16 S529-52 
[30] Gundlach C, Calabrese G, Hinder I and Martín-García J M 2005 Constraint damping in the Z4 formulation and harmonic gauge Class. Quantum Grav. 22 3767-74

[31] Rinne O and Stewart J M 2005 A strongly hyperbolic and regular reduction of Einstein's equations for axisymmetric spacetimes Class. Quantum Grav. 22 1143-66

[32] Bona C, Ledvinka T, Palenzuela C and Žáček M 2003 General-covariant evolution formalism for numerical relativity Phys. Rev. D 67104005

[33] Buchman L T and Sarbach O C A 2006 Towards absorbing outer boundaries in general relativity Preprint gr-qc/0608051

[34] Majda A and Osher S 1975 Initial-boundary value problems for hyperbolic equations with uniformly characteristic boundary Comm. Pure Appl. Math. 28 607-75

[35] Kreiss H O 1970 Initial boundary value problems for hyperbolic systems Comm. Pure Appl. Math. 23 277-98

[36] Agranovich M S 1972 Theorem on matrices depending on parameters and its applications to hyperbolic systems Funct. Anal. Appl. 6 85-93

[37] Hearn A C 2004 REDUCE User's Manual 3.8 RAND Santa Monica, CA, USA

[38] Alcubierre M et al 2004 Toward standard testbeds for numerical relativity Class. Quantum Grav. 21 589-613

[39] Boyle M, Lindblom L, Pfeiffer H P, Scheel M A and Kidder L E 2006 Testing the accuracy and stability of spectral methods in numerical relativity Preprint gr-qc/0609047

[40] Szilágyi B, Gómez R, Bishop N T and Winicour J 2000 Cauchy boundaries in linearized gravitational theory Phys. Rev. D 62104006

[41] Zink B, Pazos E, Diener P and Tiglio M 2006 Cauchy-perturbative matching reexamined: tests in spherical symmetry Phys. Rev. D 73084011

[42] Bjørhus M 1995 The ODE formulation of hyperbolic PDEs discretized by the spectral collocation method SIAM J. Sci. Comput. $16542-57$ 